\title{
ARTICLE
}

\section{The CTA1-DD adjuvant strongly potentiates follicular dendritic cell function and germinal center formation, which results in improved neonatal immunization}

\author{
Sophie Schussek ${ }^{1}$, Valentina Bernasconi ${ }^{1}$, Johan Mattsson ${ }^{1}$, Ulf Alexander Wenzel ${ }^{1}$, Anneli Strömberg ${ }^{1}$, Inta Gribonika ${ }^{1}$,
} Karin Schön ${ }^{1}$ and Nils Y. Lycke ${ }^{1}$

Vaccination of neonates and young infants is hampered by the relative immaturity of their immune systems and the lack of safe and efficacious vaccine adjuvants. Immaturity of the follicular dendritic cells (FDCs), in particular, appears to play a critical role for the inability to stimulate immune responses. Using the CD21 mT/mG mouse model we found that at 7 days of life, FDCs exhibited a mature phenotype only in the Peyer's patches (PP), but our unique adjuvant, CTA1-DD, effectively matured FDCs also in peripheral lymph nodes following systemic, as well as mucosal immunizations. This was a direct effect of complement receptor 2-binding to the FDC and a CTA1-enzyme-dependent enhancing effect on gene transcription, among which CR2, IL-6, ICAM-1, IL-1 $\beta$, and CXCL13 encoding genes were upregulated. This way we achieved FDC maturation, increased germinal center B-cell- and Tfh responses, and enhanced specific antibody levels close to adult magnitudes. Oral priming immunization of neonates against influenza infection with CTA1-3M2e-DD effectively promoted anti-M2e-immunity and significantly reduced morbidity against a live virus challenge infection. To the best of our knowledge, this is the first study to demonstrate direct effects of an adjuvant on FDC gene transcriptional functions and the subsequent enhancement of neonatal immune responses.

Mucosal Immunology (2020) 13:545-557; https://doi.org/10.1038/s41385-020-0253-2

\section{INTRODUCTION}

Protection against infection in early life is achieved through transplacental transfer of maternal lgG antibodies and secretory IgA antibodies in breast milk. ${ }^{1}$ The duration of this protection is limited to a few months after birth when the neonatal immune system is still too immature to mount an effective immune response. ${ }^{2}$ However, this immaturity also poses a major hurdle for neonatal vaccine development. $A$ focus in recent years has been to find vaccine formulations that can overcome the impaired immune responses in neonates and young infants. ${ }^{3}$ Most of this work, though, has focused on injected vaccines and much less interest has been shown in mucosal delivery, which could improve neonatal vaccination by harnessing the enhanced maturity of the local, microbiota-exposed immune system in the first few weeks of life. ${ }^{2,4}$ Speaking in favor of the latter approach is the fact that oral polio and rotavirus vaccines have both been successfully given, even to pre-term infants, with little apparent side-effects. ${ }^{5}$

The exact mechanisms underlying the immaturity of the neonatal immune system still remain to be further investigated, but it is generally agreed that intrinsic factors in the B- and T-cell compartments together with a poorly developed innate immune system are contributing elements. ${ }^{2-4}$ Indeed, a hallmark of neonates and young infants is the poor ability to develop germinal center (GC) reactions, which results in few follicular helper T cells (Tfh) and memory B cells, as well as strongly reduced isotype-switched antibody levels. ${ }^{8,9}$ A lack of performance of antigen-presenting cells (APC), in particular dendritic cells (DC), appears critically involved in the immaturity of the neonatal immune system. ${ }^{10-12}$ Furthermore, the response to pattern recognition receptor (PRR) stimulation and especially toll-like receptor (TLRs) signaling via the Myd88 adaptor protein is hampered in neonates. ${ }^{13}$ To overcome the impaired innate response to non-replicating and subunit vaccines in neonates the addition of adjuvants has been found effective in experimental models. Presently, the only widely approved adjuvants for neonatal vaccination are aluminum salts, despite their inefficacy at improving APC-functions in neonates. ${ }^{4,14}$ Therefore, the search for new adjuvants to improve neonatal vaccines is ongoing, and while some have already been licensed, more knowledge about their mechanisms of action on neonatal immune responses is critically needed. ${ }^{15,16}$

We have developed an adjuvant based on the enzymatically active CTA1-subunit of cholera toxin (CT) and a dimer of the Ddomain from Staphyloccous aureus protein $A$, the CTA1-DD adjuvant. ${ }^{17}$ In contrast to $C T$, this molecule is non-toxic and safe to use as an adjuvant, as has been well documented in mice and non-human primates. ${ }^{17,18}$ The CTA1-DD molecule is an effective mucosal and systemic adjuvant, able to stimulate a strong and balanced $\mathrm{CD}^{+}{ }^{+}$-cell response with greatly enhanced specific antibody production. ${ }^{19-21}$ A key mechanism of action is its ability to enhance GC reactions and promote development of long-lived plasma cells and memory B cells. ${ }^{19-21}$ However, how this is achieved is presently poorly known. Previous studies, have shown that CTA1-DD adjuvant activates complement and can bind to

\footnotetext{
${ }^{1}$ Mucosal Immunobiology and Vaccine Center (MIVAC), Department of Microbiology and Immunology, Institute of Biomedicine, University of Gothenburg, Gothenburg, Sweden
} Correspondence: Nils Y. Lycke (nils.lycke@microbio.gu.se)

Received: 3 June 2019 Revised: 6 December 2019 Accepted: 10 December 2019

Published online: 20 January 2020 
complement receptors 1 and 2 (CR1/CR2) on follicular dendritic cells (FDCs), and, in this way, directly affect the functions of the FDC. $^{22}$ The FDC network has a critical role in organizing B-cell follicles and the GC reaction (reviewed in ref. ${ }^{23}$ ). ${ }^{24}$ Depletion of FDC or their ability to trap immune complexes (ICs) strongly impairs class switch recombination (CSR), somatic hypermutation (SHM), and memory development in mice. ${ }^{25-27}$ Furthermore, FDCs express a number of receptor ligands and soluble factors to attract and interact with the activated B cells and provide them with integrated signals to achieve proliferation and differentiation, involving selection of high-affinity memory B cells and long-lived plasma cells. ${ }^{23,28-31}$ Of several factors, CXCL13 has been found critical for the recruitment of CXCR5-positive B cells and Th cells into the follicle to form GC. ${ }^{30,32-34}$ Although CXCL13 acts locally, it can also be detected in human plasma, where perturbations in plasma CXCL13 levels have been suggested to be a reliable biomarker for GC formation. ${ }^{35,36}$

Previously, other adjuvants, such as MF59, AS03, and AS04, have been evaluated for their ability to enhance GC and antibody responses in neonatal and infant mice. ${ }^{15,37}$ MF59 was found to promote GC reactions in infant mice primarily by promoting Tfh responses, but possibly also via an effect on innate immunity concomitant with increasing antigen availability in the lymph node. $^{15,38}$ A non-toxic, mutated version of the heat-labile enterotoxin of E.coli (LTK63) was found to enhance the GC reaction by a mechanism that was proposed to involve FDCs. ${ }^{37,39}$ Other studies have found that C-type lectin receptor (CLR) agonists were more potent than TLR-based agonists at stimulating neonatal B-cell responses. ${ }^{40}$ These authors hypothesized that the enhanced performance of CLR agonists may be due to an enhanced antigen availability for GC B cells, rather than an effect on the maturation of APCs, as seen with TLR agonists.

The present study investigated whether the CTA1-DD adjuvant could improve neonatal vaccination and evaluated if the direct targeting of this adjuvant to the FDC, via the complement receptors 1 and 2 (CR1,CD35 / CR2,CD21), was involved in the GCpromoting effect. To facilitate our studies of FDCs, we developed a mouse model in which CD21-positive cells endogenously express membrane bound GFP, whereas CD21-negative cells express tdTomato. Using this model, we could clearly distinguish pure FDCs from other stromal cell populations, which lack markers typically associated with mature FDCs. ${ }^{25,26}$ Here, we report that CTA1-DD is the first known adjuvant to bind and directly influence gene transcription in FDCs. In this way CTA1-DD greatly upregulated $\mathrm{CxCl} 13$ gene expression, which was associated with an enhanced GC reaction, following parenteral, as well as mucosal immunizations. The effect of intranasal as well as oral immunization on neonatal FDCs was compelling and these cells matured into adult-like cells that strongly promoted GC B-cell and Tfh development. Following combined oral and intranasal immunizations of neonatal mice with a candidate influenza vaccine, CTA1$3 \mathrm{M} 2 \mathrm{e}-\mathrm{DD}$, we could demonstrate that oral administration of the vaccine was effective and enhanced vaccine immunogenicity and improved protection against influenza-infection by reducing morbidity.

\section{RESULTS}

Irrespective of route of administration CTA1-DD adjuvant binds to FDCs and promotes gene expression, leading to expanded GC reactions

Following systemic administration we previously observed that the CTA1-DD adjuvant accumulated in the FDC network, a mechanism that was found to depend on complement activation and complement receptor expression. ${ }^{22}$ As mucosal membranes carry little complement, we asked if an intra nasal (i.n.) administration of the adjuvant would also accumulate in a similar manner in the draining mediastinal lymph node $(m L N)$. To this end, we monitored the kinetics of adjuvant localization in the draining lymph nodes following intravenous (i.v.), subcutaneous (s.c.), and i.n. administrations. We found that mucosal as well as systemic administration of CTA1-DD, as well as enzymatically inactive CTA1R7K-DD, resulted in co-localization of the adjuvant with $\mathrm{CD}_{3} 5^{+} \mathrm{FDC}$ in the B-cell follicle (Fig. 1a), suggesting that the DD part of the fusion protein is instrumental for FDC binding. ${ }^{22}$ As a control, mice were administered with OVA-FITC, which bound poorly to FDC when analyzed by FACS, revealing the high specificity of the CTA1-DD binding to the FDC network (Fig. 1b). Hence, the adjuvant accumulated to $\mathrm{CD} 21^{+}$FDCs after both systemic and mucosal administration and roughly $25 \%$ of the FDC had bound peak levels of the adjuvant already after $3 \mathrm{~h}$ (Fig. 1b). The augmenting effect on serum antibodies following i.n. immunization with NP-CPG was similar to that of systemic CTA1DD adjuvanted immunizations and the effect on generating increased Tfh and GC B-cell levels in the $\mathrm{mLN}$ were comparable to those seen in inguinal lymph nodes (iLN) following s.c. immunization (Fig. 1c, d; gating strategies in Supplementary Fig. 1). Taken together, the CTA1-DD adjuvant affected immune responses in draining lymph nodes similarly irrespective of the route of administration.

Next, we analyzed the consequences of CTA1-DD binding to the FDC network using the CD21-cre mouse model to drive the expression of a reporter gene (from here on referred to as $\left.\mathrm{CD}_{2} 1^{\mathrm{mT} / \mathrm{mG}}\right)$, resulting in membrane GFP-expressing $\mathrm{CD} 21^{+}$ FDCs and B cells. As expected, secondary lymphoid tissues in $\mathrm{CD} 21^{\mathrm{mT} / \mathrm{mG}}$ mice hosted B-cell follicles with a distinct FDC network (Fig. 2a upper panel). Upon immunization with CTA1-DD adjuvant, we found a much denser and higher GFP-expressing FDC (CD21) network than in unimmunized control mice, as also defined by FDC-M2 co-staining (Fig. 2a lower panel and Fig. 2b). This increase in the percentage of $\mathrm{GFP}^{+}$lymphoid stromal cells that were $\mathrm{CD}_{4}{ }^{-} \mathrm{CD}_{3} 1^{-} \mathrm{B}_{220}{ }^{-} \mathrm{F} 4 / 80^{-} \mathrm{CD}_{11 c^{-}} \mathrm{GR}^{-}{ }^{-} \mathrm{CD}_{138}^{-}$was confirmed by FACS analysis, demonstrating a significant increase in FDCs 4 days following s.c immunization with the CTA1-DD adjuvant (Fig. 2c, e, f). Furthermore, Ki67 staining of iLN sections from $C D 21^{\mathrm{mT} / \mathrm{mG}}$ mice and FACS analysis of these cells, confirmed that $\mathrm{GFP}^{+} \mathrm{FDC}-\mathrm{M2}^{+}$cells are proliferating, and increasing in percentage and number in response to CTA1-DD (Fig. 2d, f). Contrary to a previously published study using a similar CD21-Crebased model, our mice expressed CD21 (messenger RNA (mRNA) and protein; Supplementary Fig. 2a, g) in GFP ${ }^{+}$FDC, but not Cxcl12 (mRNA, Supplementary Fig. 2f) and we did not find labeled CD21 $21^{-}$stromal cells in the T-cell zone, as reported (Fig. 2a and Supplementary Fig. 2g). ${ }^{41,42}$ To further evaluate our CD21 $\mathrm{mT} / \mathrm{mG}$ model, we undertook an extensive FACS- and quantitative PCR (qPCR) analysis of FDC and non-FDC stromal cells (Supplementary Fig. $2 a-f) .{ }^{28,41,43}$ Focusing on GC relevant genes, we selected the stromal population expressing the highest levels of the $\mathrm{CxCl13}$ for further analysis as this encodes the main factor responsible for attracting activated Tfh and B cells to the GC. ${ }^{30,32-34}$ Quantitative reverse transcription PCR (RT-qPCR) analysis of sorted $\mathrm{GFP}^{+}$ lymphoid stromal subpopulations showed highest gene expression of $\mathrm{Cr} 2$ and $\mathrm{CxCl} 13$ in FDC-M2 stained cells, affirming a unique FDC-identity of these cells in our CD21 $1^{\mathrm{mT} / \mathrm{mG}}$ mice (iLN; Supplementary Fig. $2 \mathrm{f}$ and spleen; Supplementary Fig. $2 \mathrm{~h}$ ), and allowing for a detailed investigation of the impact of vaccineadjuvant treatment on this critical subset of cells for the GC reaction (Fig. 2e).

Next, we investigated whether the CTA1-DD adjuvant could directly affect the function of FDCs. To this end, we immunized $\mathrm{CD} 21^{\mathrm{mT} / \mathrm{mG}}$ mice s.c. with CTA1-DD adjuvant or its enzymatically inactive mutant, CTA1R7K-DD, and isolated FDC-M2 ${ }^{+}$GFP $^{+}$FDC from the iLNs 4 days after immunization in agreement with already well established protocols. ${ }^{32,44}$ After enzymatic digestion of draining LNs, FDCs were identified as shown in representative FACS plots (Supplementary Fig. 2b). For gene expression 


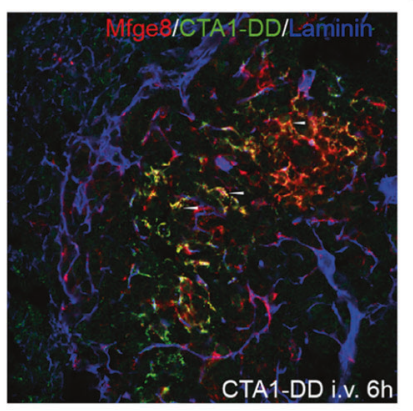

i.n. $\mathrm{mLN}$

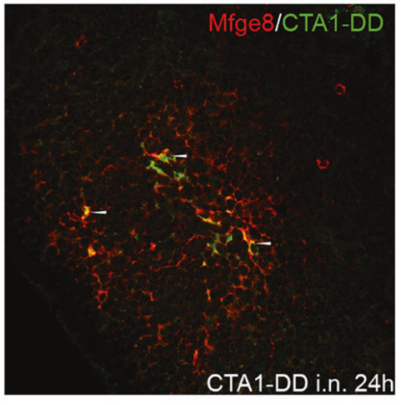

i.v. spleen

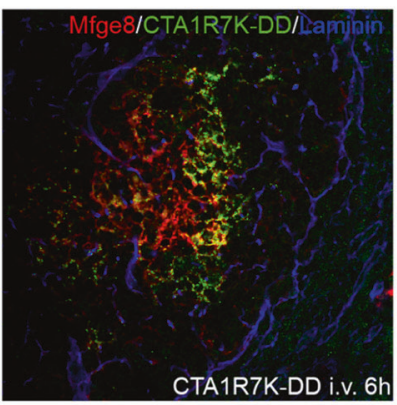

S.c. iLN

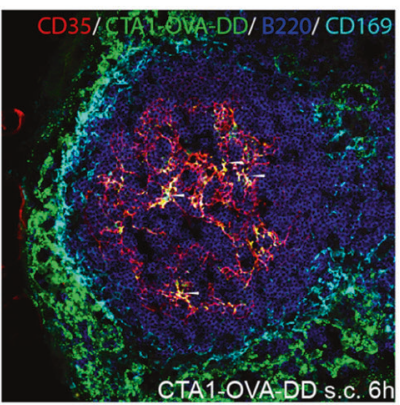

b

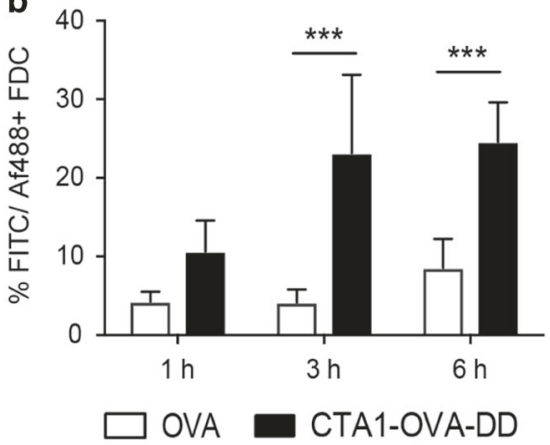

c serum IgG anti-NP titer

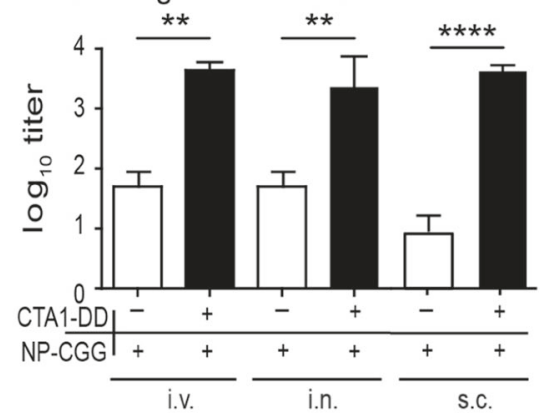

d

i.n. $\mathrm{mLN}$

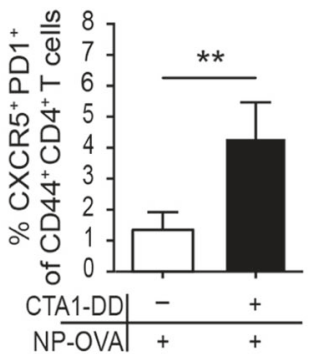

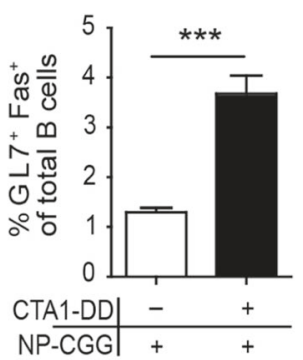

S.c. iLN

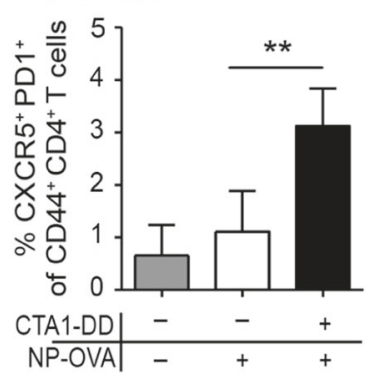

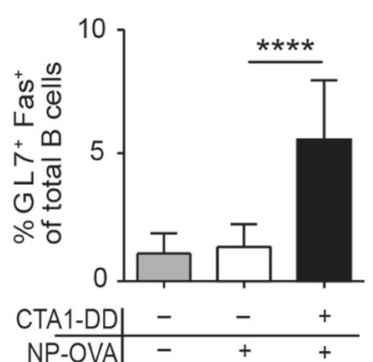

Fig. 1 The CTA1-DD adjuvant given through multiple routes targets the FDC network and enhances the GC reaction in draining lymph nodes. a Microscopy sections from indicated tissues showing deposition of CTA1-DD or CTA1R7K-DD adjuvant at $6 \mathrm{~h}$ (i.v. and s.c.) or $24 \mathrm{~h}$ (i.n.) following immunization via different routes. FDC (Mfge8 or CD35, red), and CTA1-DD (green) were identified with laminin (blue) in spleen and B220 (blue) plus CD169 (cyan) in iLN. All images were taken with an inverted confocal microscope (LSM700) with x20 magnification. The size bars indicate $100 \mu \mathrm{m}$. These are representative sections from individual mice in groups of three mice showing similar results. $\mathbf{b}$ FACS analysis of antigen binding to FDC following immunizations with OVA-FITC alone or incorporated in the CTA1-OVA-DD fusion protein conjugated to AF488 and containing an equimolar dose $(5 \mu \mathrm{g})$ of OVA-peptide. c IgG anti-NP-specific serum antibodies were determined by ELISA and given as $\log _{10}$-titers \pm SD after 2 i.v., s.c., or 3 i.n. immunizations as in $\mathbf{a}$ and $\mathbf{b}$. These are pooled data from three experiments ( $n=3$ in each group). d The percentage of TFH cells (CD4 ${ }^{+}$FoxP3 ${ }^{-}$CXCR5 ${ }^{+}$PD- $1^{+}$) or activated B cells $\left(\mathrm{GL}_{-1} 7^{+} \mathrm{Fas}^{+} \mathrm{IgD}^{-}\right.$of all CD19 ${ }^{+}$) in the GC in the mediastinal $\mathrm{LN}$ $(\mathrm{mLN})$ (left panel) or inguinal LN (iLN) (right panel) was assessed by FACS 10 days following an i.n. (mLN) or s.C. (iLN) immunization with $5 \mu \mathrm{gg}$ NP-OVA with (black bars) or without (white bars) $10 \mu \mathrm{g}$ admixed CTA1-DD adjuvant, as indicated. Cells from naive unimmunized mice (light gray bars) were included as controls (right panel). Statistical significance was determined by one-way ANOVA followed by Tukey correction for multiple comparisons; ${ }^{*} p<0.05,{ }^{* *} p<0.01,{ }^{* * *} p<0.001,{ }^{* * * *} p<0.0001$.

analysis, we isolated mRNA and conducted RT-PCR for preselected genes using custom-made qPCR plates (Qiagen and confirmed with qPCR primers). Several genes were upregulated in iLN FDCs after s.c. immunization with CTA1-DD adjuvant (Fig. 2g). Most importantly, Cxcl13 gene expression was increased ninefold compared to resting/naive FDCs, suggesting a direct effect on the ability to recruit activated lymphocytes to the FDC network (Fig. 2g). Other genes influencing GC formation and maintenance were also significantly upregulated, including Icam-1 (26-fold), Cr2 (14-fold), II-6 (sixfold) and to a lesser extent $I-1 \beta, C d 40, L t \beta r$, and Baff encoding genes (Fig. $2 \mathrm{~g}$ ). The observed upregulation of FDC gene expression was enzyme-dependent, because the inactive CTA1R7K-DD molecule had only minimal effects, especially on the expression of $\mathrm{CxCl13}$, II-6 and Icam-1 encoding genes. By contrast, the mutant CTA1R7K-DD caused a significant increase in $1 /-15$ gene expression (25-fold higher than naive and 2.5-fold higher than the CTA1-DD adjuvant), indicating that also CTA1R7K-DD was biologically active, but having a differential effect on gene transcription compared to the enzymatically active CTA1-DD molecule (Fig. 2g). Noteworthy, adjuvant activation of gene expression in other lymphoid stromal cell populations $(C x C l 12, V c a m-1)$ or indicating contamination with GC B cells $(\mathrm{BCl}-6)$, were not apparent after immunization with CTA1-DD or its inactive mutant (Fig. 2g). Neither were genes involved in putative FDC differentiation, such as Rank- $L$ and Madcam-1, affected by adjuvant treatment (Fig. 2g). The enhancing effect on $\mathrm{CxCl13}, \mathrm{Cr} 2$ and Icam-1 gene expression was observed already on day 1 after immunization with the CTA1-DD adjuvant, increased to day 4 and was sustained still after 6 days (Fig. 2h). 
a
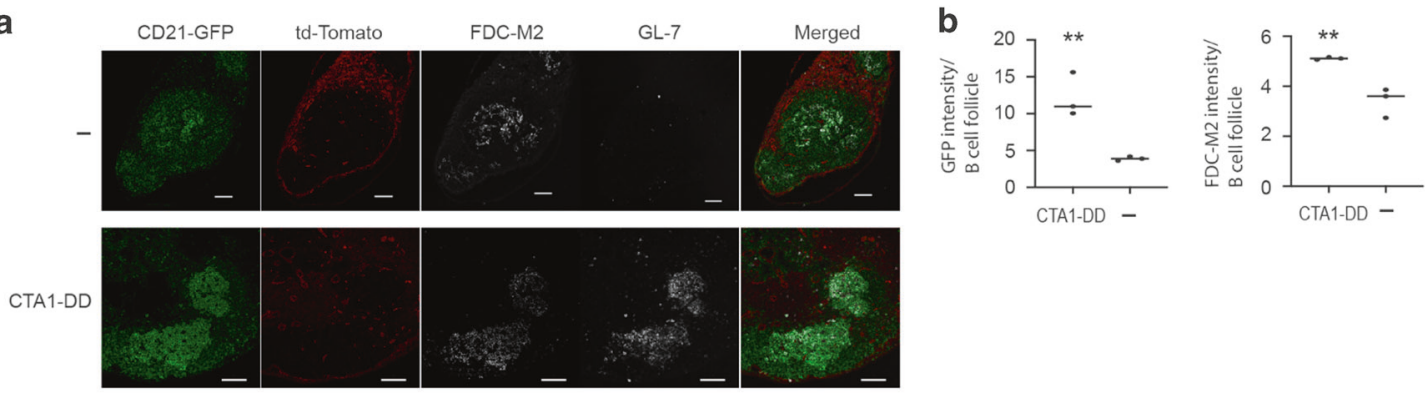

C
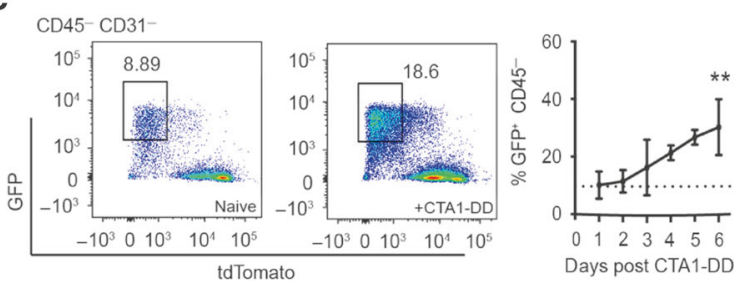

d
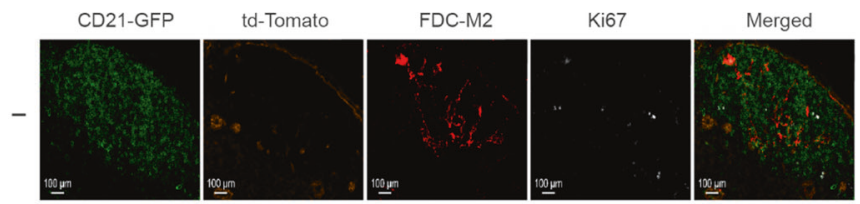

e
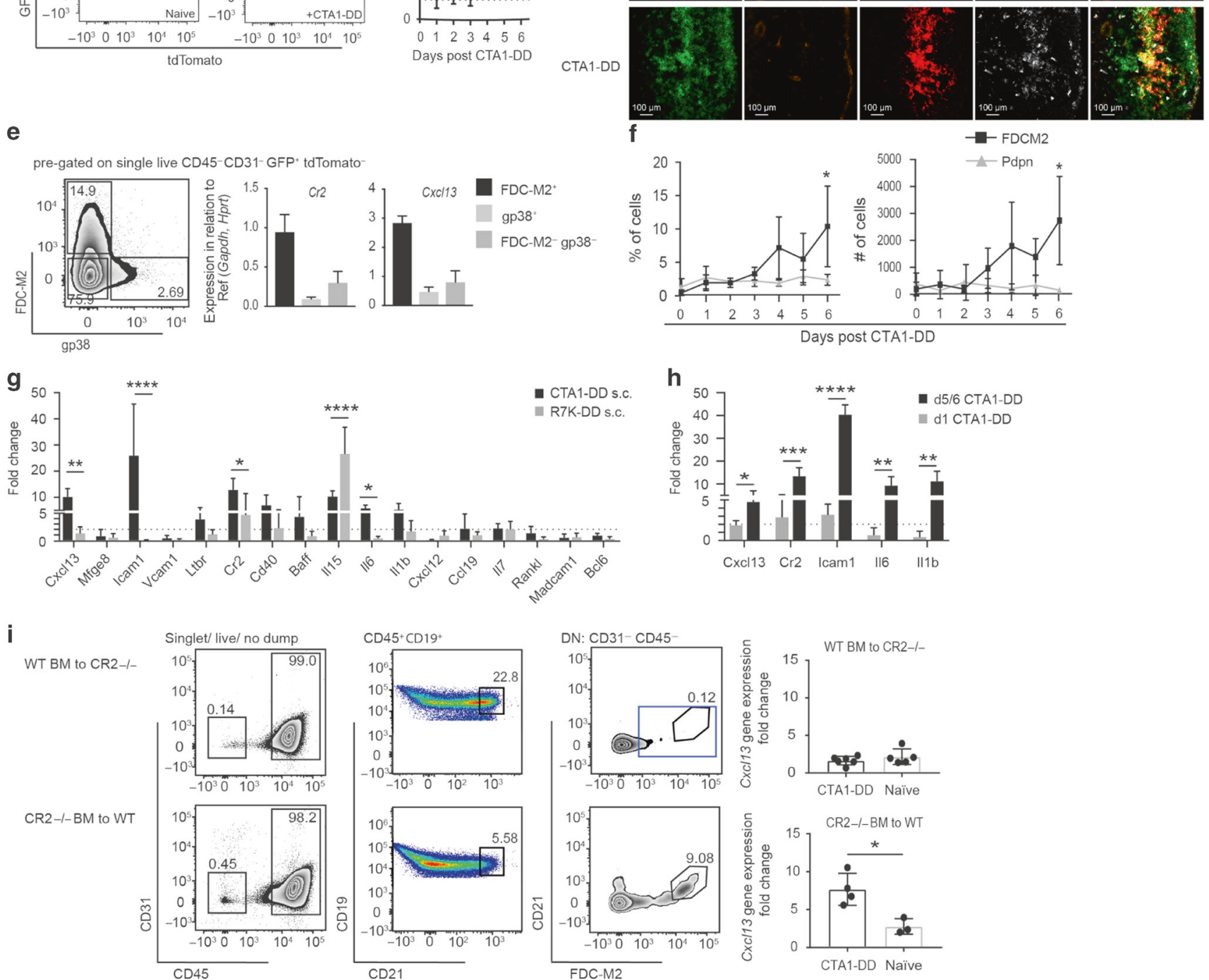

To test the notion that these gene transcriptional effects of CTA1DD were secondary to binding to the CR2 on the FDCs and to exclude any impact of gene transcription in potential contaminating $B$ cells in our analysis, we generated bone marrow (BM) chimeras by transferring wild-type (WT) BM to CR2-deficient mice or vice versa. We found that in mice with CR2 expression on $B$ cells only or in mice where FDCs were CR2 deficient (WT BM to CR2 -/- chimeras), expression of target genes was unchanged, while in mice with CR2 replete FDCs we found a significant upregulated $\mathrm{Cxcl13}$ gene expression (Fig. 2i). Hence, the enhancing effect on gene transcription of CTA1-DD adjuvant was linked to CR2 expression on FDCs and unaffected by $B$ cells irrespective of CR2 expression. Taken together, our data provide compelling evidence that CTA1-DD adjuvant not only targets FDCs via CR2, but also directly modulates FDC function by affecting gene expression at an early time point, promoting GC formations, as well as over time supporting a strong GC reaction. 
Fig. 2 CTA1-DD adjuvant treatment results in expansion and enhanced gene expression in targeted FDCs; a CD21 ${ }^{\mathrm{mT} / \mathrm{mG}} \mathrm{mouse} \mathrm{model}$ for isolating GFP ${ }^{+}$stromal cells. a Representative sections of iLN from CD $21^{\mathrm{mt} / \mathrm{mG}}$ naive mice (upper panel) or 12 days following a s.c. immunization with $10 \mu \mathrm{g}$ CTA1-DD adjuvant (lower panel). Sequential tissue sections were stained for the FDC network (FDC-M2 ${ }^{+}$) or GC $\left(\mathrm{GL}-7^{+}\right)$; merged pictures show the overlap of CD21-GFP with FDC-M2. All images were taken with an inverted confocal microscope (LSM700) with $\times 20$ magnification. The size bars indicate $100 \mu \mathrm{m}$. These are representative sections from individual mice in groups of three mice showing similar results. b Intensity of GFP expression or FDC-M2 staining in tissue sections was measured using Fiji and is shown as color intensity per B-cell follicle for naive or CTA1-DD immunized mice $(n=3)$. c FACS analysis of CD45 ${ }^{-}$CD31- $\mathrm{LN}$ cells from naive or CTA1-DD immunized $\mathrm{CD} 21^{\mathrm{mT} / \mathrm{mG}}$ mice at 4 days (left panels) or the kinetics (right panel) of FDC responses after immunization. Representative panels of two experiments with $\mathrm{n}=3$ mice each and statistical significance $(* * p<0.01)$ using one-way ANOVA of the effect of the adjuvant treatment on FDC frequencies compared to those observed in untreated mice (dotted line). d Representative sections of iLN from naive CD2 $11^{\mathrm{mT} / \mathrm{mG}} \mathrm{mice}$ (upper panel) or 12 days following a s.c. immunization with $10 \mu \mathrm{g}$ CTA1-DD adjuvant (lower panel). Tissues from three mice per group were stained for FDC network (FDC-M2 ${ }^{+}$) and cell proliferation was assessed by Ki67-staining; merged pictures show the overlap of CD21-GFP with FDC-M2 ${ }^{+}$and $\mathrm{Ki}^{+} 7^{+}$cells. e Validation of FDC status was done by $\mathrm{Cr} 2$ and $\mathrm{CxCl} 13$ gene expression analysis of sorted GFP ${ }^{+} \mathrm{FDC}_{-\mathrm{M} 2}{ }^{+}, \mathrm{GFP}^{+}$ $\mathrm{gp}^{+} 8^{+}$or GFP ${ }^{+}$FDC-M2 gp38 $^{-}$(DN) cells from naive CD21 ${ }^{\mathrm{mT} / \mathrm{mG}}$ mice. Data shown for $n=6$ mice pooled from two experiments as relative gene expression to the reference genes Gapdh and Hprt. f Kinetics of the percentage and number of FDC-M2 $2^{+}$or Pdpn $^{+}$cells of GFP ${ }^{+} \mathrm{CD}_{4}{ }^{-}$ CD31 ${ }^{-}$tdTomato $^{-}$cells in iLN following s.c. immunization with $10 \mu \mathrm{g}$ CTA1-DD adjuvant of CD21 ${ }^{\mathrm{mT} / \mathrm{mG}}$ mice $(n=6$ pooled from two experiments). $\mathbf{g}$ Gene expression analysis using qPCR of mRNA from FDCs isolated from iLN 4 days after a s.c. immunization with $10 \mu \mathrm{g}$ of CTA1-DD adjuvant. $\mathbf{h}$ Kinetics of gene expression in FDCs in iLN revealing early (day 1 ) and late (days 5/6) changes following s.c. immunization with $10 \mu \mathrm{g}$ of CTA1-DD adjuvant as compared to gene expression in FDCs from naive control mice. For $\mathbf{g}$ and $\mathbf{h}$, results are given as the mean fold-change \pm SD of two pooled independent experiments ( $n=3$ in each group) relative to the mRNA levels of each gene observed in samples from unimmunized control mice $\left(2^{\mathrm{ddct}}\right)$. The dotted line indicates a twofold change. Statistical significance relative to controls was assessed using repeated-measures ANOVA with Sidak correction for multiple measurements; ${ }^{*} p<0.033,{ }^{* *} p<0.002,{ }^{* * *} p<0.0002,{ }^{* * * *} p<0.0001$. i Chimeric mice were made through transplantation of WT BM to irradiated CR2 $2^{-/-}$mice (upper panel) or CR2 $2^{-/-}$BM transplantation to irradiated WT mice (lower panel), thus, reflecting an absence of CR2 in either FDCs (upper) or B cells (lower), respectively. The CR2 ${ }^{-1-}$ chimeric mice were immunized s.C. with or without $10 \mu \mathrm{g}$ CTA1-DD adjuvant and 4 days later FDCs were isolated and $C x c / 13$ gene expression was assessed using qPCR in CTA1-DD adjuvant-treated FDCs as compared to FDCs from naive control mice. Unless otherwise stated statistical significance was determined by one-way ANOVA followed by Tukey correction for multiple comparisons; ${ }^{*} p<0.05,{ }^{* *} p<0.01,{ }^{* * *} p<0.001,{ }^{* * * *} p<0.0001$.

CTA1-DD accelerates FDC network maturation and enhances GC responses in neonates

It is well documented that newborn mice do not develop GC because they lack mature FDCs. ${ }^{45,46}$ To further explore the potential of the $C D 21^{\mathrm{mT} / \mathrm{mG}}$ model system, we investigated young neonatal mice and compared the $\mathrm{GFP}^{+}$FDC network in young and adult mice with or without immunization with CTA1-DD adjuvant. We found that in 7-day-old mice, no $\mathrm{GFP}^{+} \mathrm{CD} 35^{+}$FDCs could be identified in the spleen or peripheral lymph nodes, ${ }^{24,47}$ albeit CD21-GFP ${ }^{+}$cells were clearly seen in the $\mathrm{B}_{220^{+}} \mathrm{B}$ cells (Fig. 3a). By contrast, we observed clear evidence of an FDC network in Peyer's patches (PPs), supporting the hypothesis that microbial colonization of the gut in early life drives maturation of FDC maturation $^{48,49}$ (Fig. 3a). However, at 17 days of age, adult levels of FDC-networks $\left(\mathrm{CD} 35^{+}\right)$were observed also in peripheral lymph nodes of $\mathrm{CD} 21^{\mathrm{mT} / \mathrm{mG}}$ mice (Fig. 3a). ${ }^{24,47}$ We then asked whether the direct gene transcriptional effect of the CTA1DD adjuvant on FDCs could be used to improve GC responses also in neonatal mice. Indeed, we found that $\mathrm{CD} 21^{+} \mathrm{CD} 45^{-}$FDCs isolated 4 days after CTA1-DD immunization on post-natal day 3 showed strongly upregulated $\mathrm{CxCl13}$ gene expression as compared to 7-day-old mice exhibiting no FDCs or unimmunized naive FDC at 17 days of age (Fig. 3b). We found newly developed FDC-networks in 10-12\% of B-cell follicles of the CTA1-DD immunized mice (Fig. 3c). Most impressively, we observed mature $\mathrm{GC}\left(\mathrm{GL}-7^{+}\right)$in lymph node sections that were comparable to those in adult adjuvant-treated mice 12 days following a s.c. immunization with NP-OVA admixed with CTA1-DD (Fig. 3d). Indeed, neonatal mice developed the same frequency of GC per B-cell follicle as adult mice as evaluated by $I H C$, using the same immunization regimen (Fig. 3e). Importantly, antigen-specific isotype-switched anti-NP antibodies were significantly enhanced in neonatal mice following a single s.c. immunization with CTA1-DD adjuvant compared to mice given NP-OVA antigen alone (Fig. 3f). This antigen-specific antibody response increased over time even without a booster immunization, indicating persistence of neonatally induced plasmablasts for at least 5 weeks after priming (Fig. 3f). A booster immunization further elevated the adjuvanted response in neonatal mice to a level comparable to that seen in adult mice (Fig. $3 f$ compare to adult levels in Fig. 1c). We concluded that CTA1-DD adjuvant enhanced $\mathrm{Cxc} / 13$ gene transcription and effectively matured neonatal FDCs, which also resulted in strongly enhanced isotype-switched antibody responses. In fact, the adjuvant fully compensated the normally poor ability of neonatal mice to respond to vaccination and significantly increased frequencies of GC B cells and TFH cells both after prime and boost immunizations (Fig. 4a-d). A linear regression analysis indicated that the increased frequencies of TFH and GC B cells correlated equally well in adult and neonatal mice, confirming the exceptional effectiveness of the CTA1-DD adjuvant (Fig. 4e). This was also observed as a dramatically increased expression of $\mathrm{BCL}^{+} \mathrm{TFH}$ cells after CTA1-DD adjuvant treatment of neonatal mice (Fig. 4f).

CTA1-DD adjuvant strongly enhances Tfh and GC B-cell responses in the Peyer's patches following oral immunization

The FDC network in neonatal CD21 $\mathrm{mT} / \mathrm{mG}$ mice appeared immature in the spleen and most peripheral lymph nodes except for in the small intestine where PPs exhibited distinct FDC network formations. This gave us the idea to explore this site further in our attempts to successfully vaccinate neonatal individuals. ${ }^{50,51}$ Hence we turned to per oral (p.o.) immunizations, although we had previously experienced failure of CTA1-DD to adjuvant oral vaccination in adult mice. ${ }^{52}$ We immunized CD21 $1^{\mathrm{mT} / \mathrm{mG}}$ mice at 5 days of age by feeding them NP-OVA with or without CTA1-DD adjuvant and boosted the mice via oral gavage with NP-OVA alone after 30 days. The induction of TFH and GC B cells was assessed in the PPs, as well as the draining sub-mandibular $L N(s m L N)$ and the mesenteric LN (MLN). We found strikingly enhanced immune responses in mice given oral CTA1-DD adjuvant with significantly increased frequencies of GC B cells and TFH cells both after initial priming and after a boost immunization in the PP, in particular (Fig. 5a-d). Indeed, in the PP, $60 \%$ of the $\operatorname{lgD}{ }^{\text {low }} \mathrm{CD} 95^{+} \mathrm{GL} 7^{+} \mathrm{B}$ cells in adjuvant-treated mice expressed Ephrin-B1, a reliable marker of GC B cells, as opposed to $30 \%$ in unadjuvanted mice ${ }^{53}$ (Fig. 5e). We also noticed a higher frequency of memory $\mathrm{CD}^{+} 3^{+} \mathrm{B}$ cells (Fig. 5e), as well as plasma cells $\left(\mathrm{CD} 138^{+} \mathrm{CD} 19^{+}\right.$cells; Fig. $\left.5 f\right)$ in the PPs following oral CTA1-DD treatment of neonatal mice. ${ }^{54-56}$ These changes in the PP GC environment accompanied a significantly stronger serum antigen-specific lgG response and high-affinity antibody titers as assessed by the ratio of NP4/NP30 
a

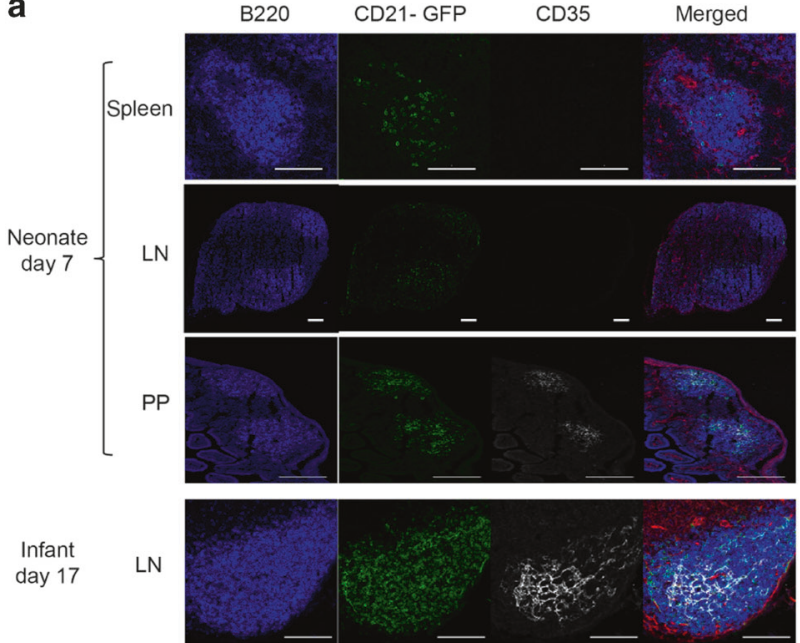

b

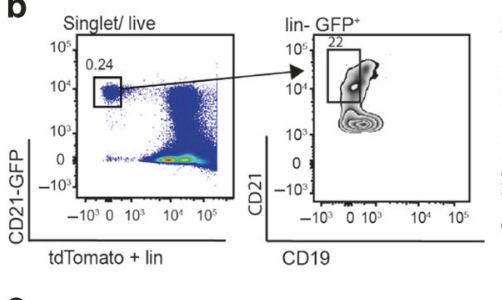

C iLN of 1 week-old CD21-cre $\mathrm{mT} / \mathrm{mG}$

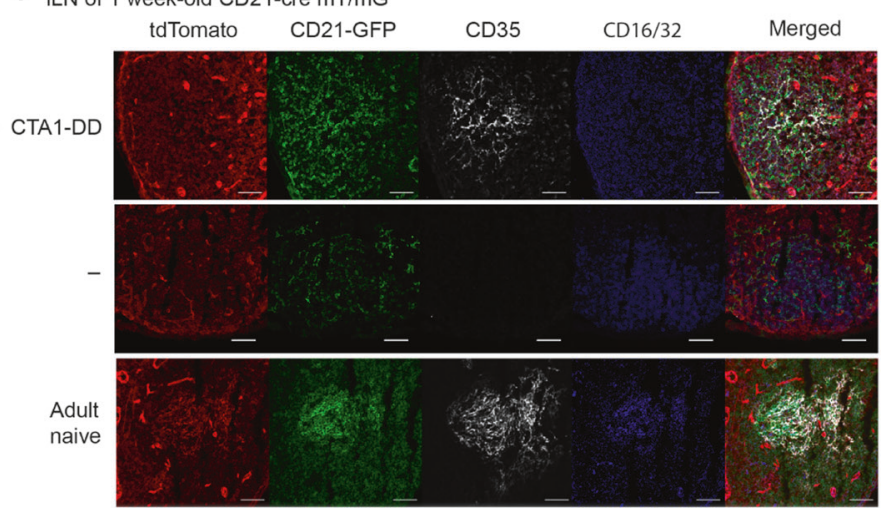

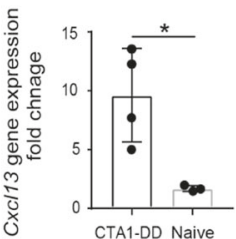

CTA1-DD Naive d

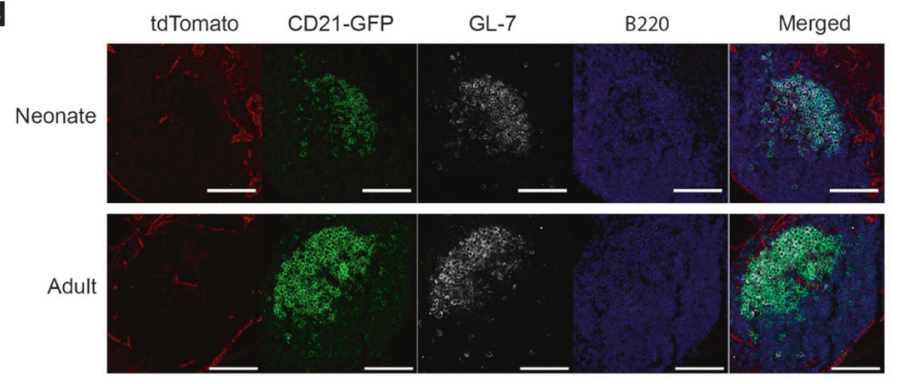

e

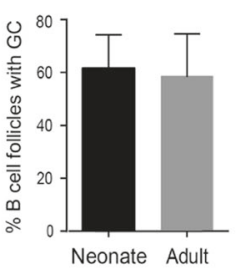

f

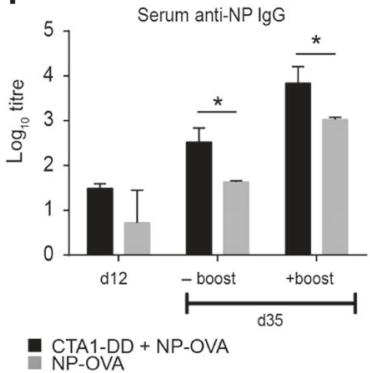

Fig. 3 CTA1-DD adjuvant accelerates FDC expansion and maturation also in neonatal draining lymph nodes. a Tissue sections from spleen, iLN, or PP from unimmunized naive neonatal (7-day-old) or infant (17-day-old) CD21 ${ }^{\mathrm{mT} / \mathrm{mG}}$ mice. Tissues were stained for B-cell follicles $\left(\mathrm{B}_{22}{ }^{+}\right.$, blue) and FDC (CD35 ${ }^{+}$, white). The size bars indicate $100 \mu \mathrm{m} . \mathbf{b} \mathrm{CD} 21^{\mathrm{mT} / \mathrm{mG}}$ mice were immunized s.c. with $10 \mu \mathrm{g}$ CTA1-DD on postnatal day 3. FDC (GFP ${ }^{+} \mathrm{CD} 21^{+} \mathrm{CD}^{-} 9^{-} \mathrm{CD} 45^{-} \mathrm{CD} 31^{-}$tdTomato') were sorted 4 days after immunization (post-natal day 7) and $\mathrm{CxC} / 13$ gene expression in FDC from iLN was assessed in CTA1-DD immunized neonate mice and compared to that observed in naive 17-day-old mice. Results are given as the mean fold-change \pm SD $\left(2^{\text {ddCt }}\right)$ and statistical significance was determined by student's $t$-test, ${ }^{*} p<0.05$. $c$ Maturation of neonatal FDCs was assessed in tissue sections from iLN from 7-day-old CD21 ${ }^{\mathrm{mT} / \mathrm{mG}}$ mice with or without a s.c. immunization with $10 \mu \mathrm{g}$ CTA1DD adjuvant given 4 days before. Representative microtome sections; red: tdTomato, green: CD21-GFP, white: CD35, blue: CD16/32. The size bars indicate $50 \mu \mathrm{m}$. d, e, f CD21 ${ }^{\mathrm{mT} / \mathrm{mG}}$ mice were immunized s.c. with $5 \mu \mathrm{g}$ NP-OVA with or without $10 \mu \mathrm{g}$ CTA1-DD on post-natal day 6 . d Tissue sections of iLN from CTA1-DD adjuvant immunized neonatal (post natal on day 6) or adult CD21 ${ }^{\mathrm{m}} / \mathrm{mG}$ mice and analyzed 12 days later for GC reactions by labeling for B220 (blue) and GL-7 (red). The size bars indicate $100 \mu \mathrm{m}$. e The frequency of B-cell follicles containing a GC was determined as the mean \pm SD from ten sequential sections of iLN from three mice in each group. All images were taken with an inverted confocal microscope (LSM700) with x20 magnification. f Serum levels of IgG anti-NP $\log _{10}$ titers were determined by ELISA after 12 and 35 days following a priming and 5 days following a booster immunization on day 30 , respectively. A statistical analysis was performed using two-way ANOVA followed by Dunnet correction for multiple comparison; ${ }^{*} p<0.05$.

titers, in particular, were greatly augmented, indicating that somatic hypermutation was promoted in neonatal PPs following oral CTA1-DD adjuvant treatment (Fig. 5g). Local gut mucosal $\lg A$ expressing B-cell populations were also significantly increased following p.o. priming with CTA1-DD adjuvant in neonatal mice (Fig. 5h). Thus, in contrast to adult mice (Fig. 5i), oral priming immunizations, even with low doses of antigen, revealed a strong adjuvant effect of CTA1-DD in neonatal mice.

Oral immunization of neonates with a candidate influenza vaccine strongly reduces morbidity against a live challenge infection To assess the protective potential of an influenza virus vaccine formulation based on the CTA1-DD platform, we primed neonatal mice orally with CTA1-3M2e-DD at the age of 5-7 days. This was followed by two CTA1-3M2e-DD booster immunizations given i.n. before we evaluated the protective effect against a live challenge infection with the $\mathrm{X} 47$ influenza virus strain (a reassortant of $\mathrm{A} /$ Victoria/3/75 (H3N2) and A/Puerto Rico/8/34(H1N1)). We detected substantial increases in serum anti-M2e lgG-specific antibody levels in mice that received the oral priming dose compared to i.n. only immunized mice (Fig. 6a). In fact, the serum titers were tenfold increased in mice that had received oral priming with the adjuvant compared to i.n. immunized mice (Fig. 6a). Virus-challenged mice were monitored for weight loss, morbidity and mortality for 15 days of the infection. The orally primed neonatal mice exhibited a reduced morbidity and recovered significantly faster than i.n only immunized mice (Fig. 6b). These mice gained weight better upon recovery from the infection. Despite this the overall survival rate was 50 to $60 \%$ in both groups compared to the phosphate buffered saline (PBS) control mice, which all died from the lethal challenge infection (Fig. 6b). Also, M2e-tetramer-specific lung CD4 ${ }^{+}$ $T$ cells were effectively induced in both orally primed and i.n. only 
$\mathbf{a}_{\text {Representative FACS plots: GC B cells }}$

Neonate

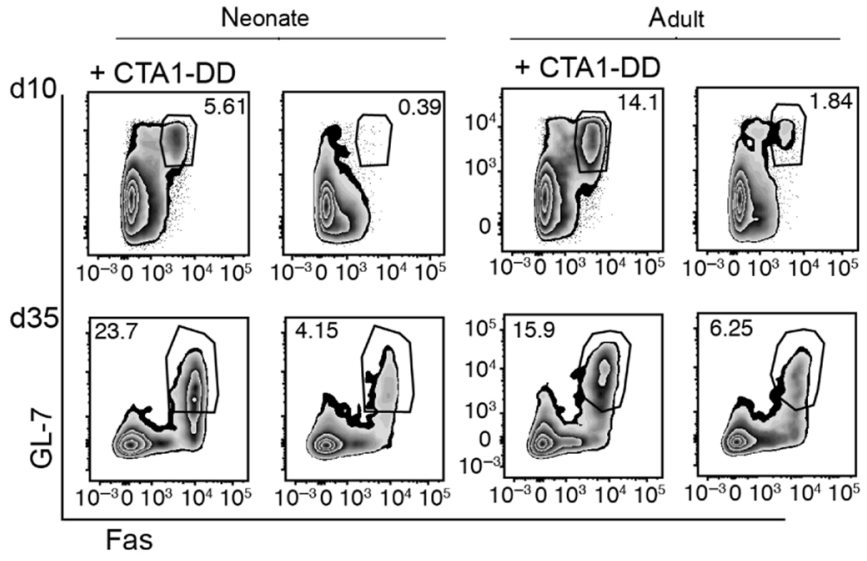

C Representative FACS plots: TFH

Neonate
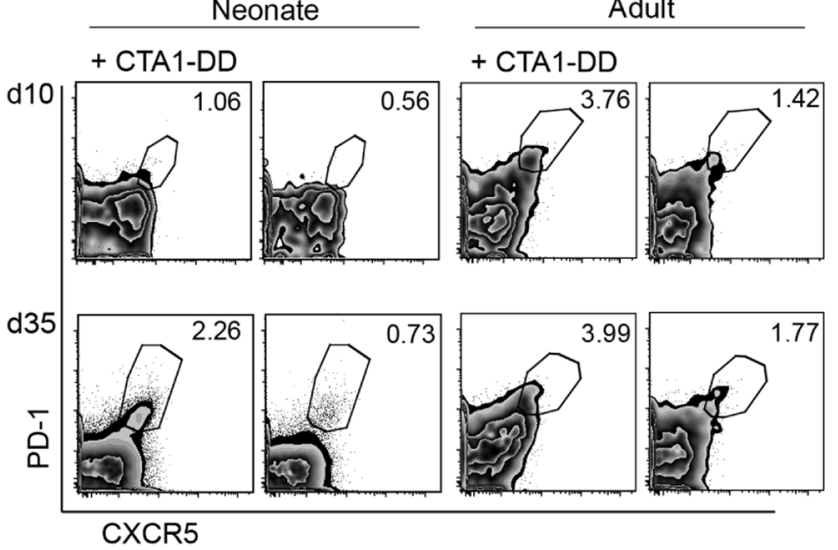

b CTA1-DD mediated GC B cells induction d10
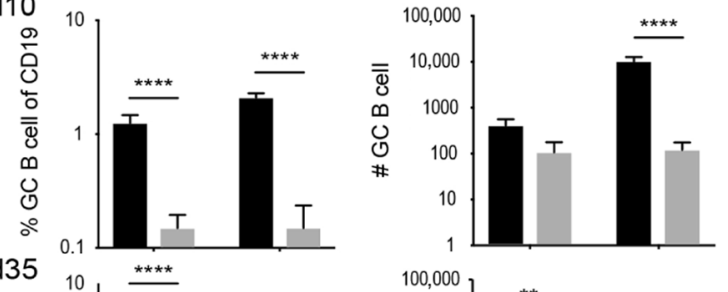

d35
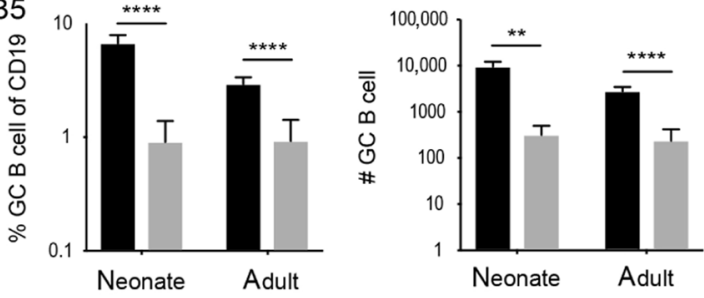

d CTA1-DD mediated TFH induction

CTA1-DD NP-OVA NP-OVA

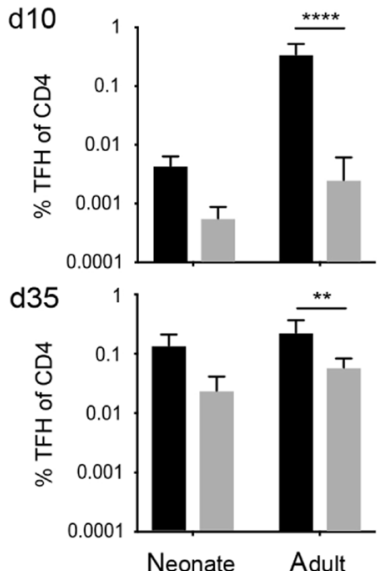

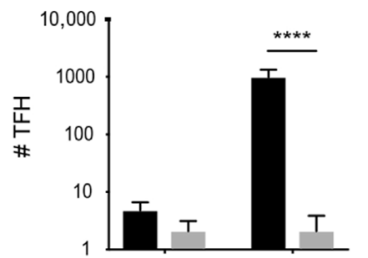

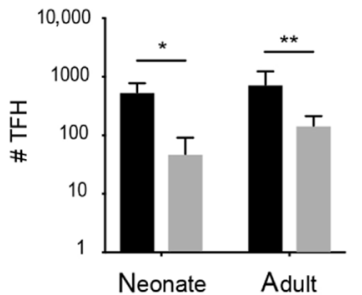

e Correlation of GC B cell with TFH frequencies Neonate d10 d35

\begin{tabular}{ll} 
Adult & \\
\hline $\mathrm{d} 10 \quad \mathrm{~d} 35$
\end{tabular}
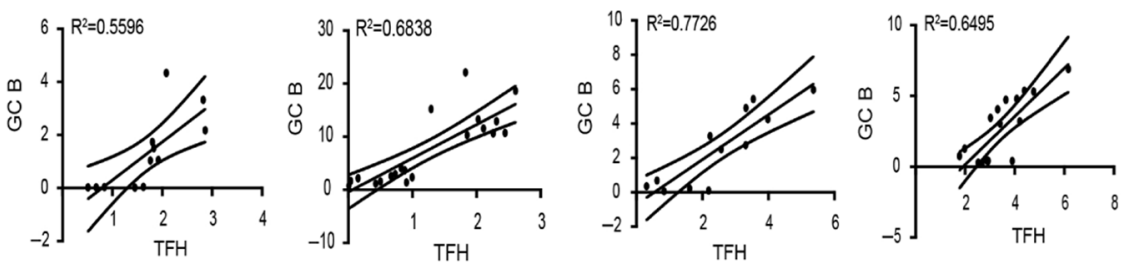

f $\mathrm{Bcl}-6$ expression in TFH

Fig. 4 Effects of CTA1-DD adjuvant on germinal center responses in neonatal mice. Adult and neonatal (5-7-days-old) mice were immunized with $5 \mu \mathrm{g}$ NP-OVA alone (gray) or admixed with $5 \mu \mathrm{g}$ CTA1-DD (black) s.c. and boosted at d30 post prime with $5 \mu \mathrm{g}$ NP-OVA s.C. a Representative FACS plots of GC B cells (CD19 ${ }^{+} \mathrm{IgD}^{\text {lo }} \mathrm{CD} 38^{\mathrm{lo}} \mathrm{Fas}^{+} \mathrm{GL}-7^{+}$) in the iLN following immunization with the indicated frequency of Fas $^{+} \mathrm{GL}^{+}$cells in the gated population of $\mathrm{IgD}^{\mathrm{lo}} \mathrm{CD} 38^{\mathrm{lo}} \mathrm{B}$ cells. $\mathbf{b}$ The frequency and absolute number of $\mathrm{GC} B$ cells of total CD19 $9^{+} \mathrm{B}$ cells in the iLN of neonatal and adult mice on day 10 post prime (upper panel) or day 5 post boost (d.35, lower panel). c Representative FACS plots of TFH cells $\left(\mathrm{CD}^{+} \mathrm{Foxp}^{-} \mathrm{CD} 44^{+} \mathrm{CXCR5^{+ }}{ }^{\mathrm{PD}} 1^{+}\right)$in the iLN following immunization with the indicated frequencies of CXCR5 ${ }^{+} \mathrm{PD} 1+\mathrm{cell}$ in the

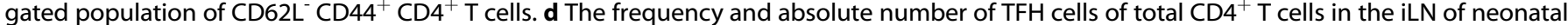
and adult mice on day 10 post prime (upper panel) or day 5 post boost (d.35, lower panel). e Linear regression analysis of the correlation between the frequency of TFH and GC B cells in CTA1-DD immunized neonatal mice as compared to that found in adult mice after s.c priming (d.10) or booster (d.35) immunizations. $\mathbf{f}$ An increase in Bcl-6 expression in TFH cells in response to CTA1-DD adjuvant is given as the ratio of MFI levels exhibited by TFH over non-TFH CD4 T cells in neonatal mice following a priming immunization (day 10) or after a booster dose (day 35). For $\mathbf{b}$, $\mathbf{d}$, and $\mathbf{f}$ statistical analysis was performed using two-way ANOVA followed by Dunnet correction for multiple comparison; ${ }^{*} p<0.05$, ${ }^{* *} p<0.01,{ }^{* * * *} p<0.0001$.

immunized mice as observed after the challenge infection (Fig. 6c). Similarly, comparable M2e-specific serum IgG and BAL IgA were recorded in both groups after infection (Fig. 6d). Surprisingly, the total gut IgA antibody levels in intestinal lavage after the challenge infection were comparable between the two groups, suggesting that the local intestinal priming effect of oral immunization appeared ineffective and no M2e-specific antibodies were detected (Fig. 6d, e, not shown). Importantly, the enzymatically inactive fusion protein CTA1R7K-3M2e-DD did not stimulate M2especific serum lgG or $\lg A$ responses nor did it induce M2e-specific 
a $\mathrm{GC} B$ cell responses in PP after priming
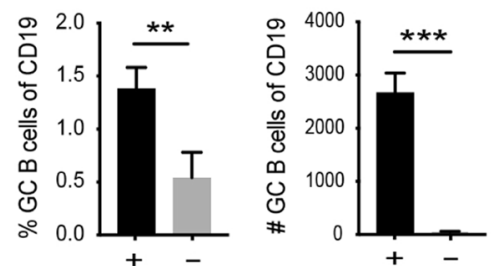

C TFH responses in PP after priming
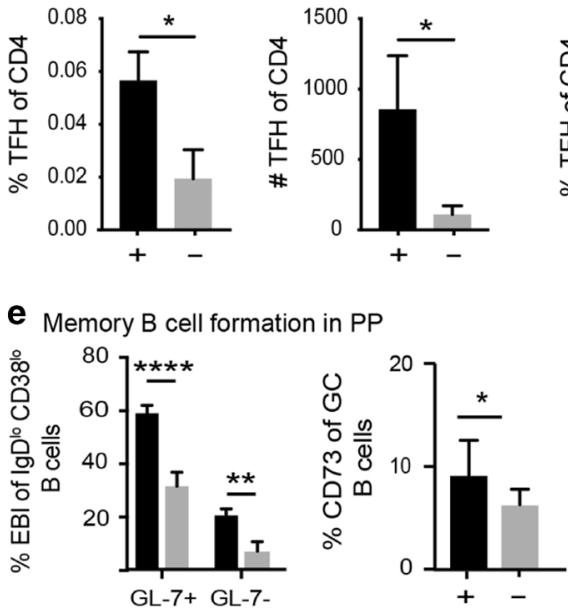

9 NP specific antibody titres

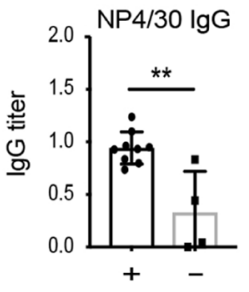

$\mathbf{h}_{\lg A+G C B \text { and plasma cells }}$
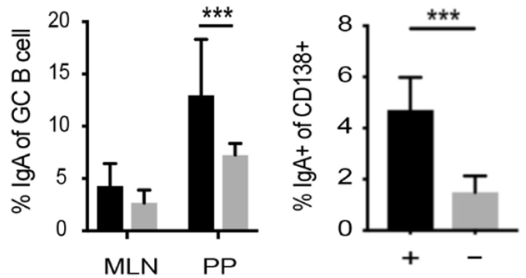

Plasma cell formation
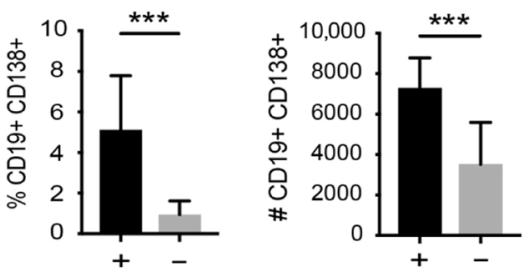

i p.o. CTA1-DD in adult mice
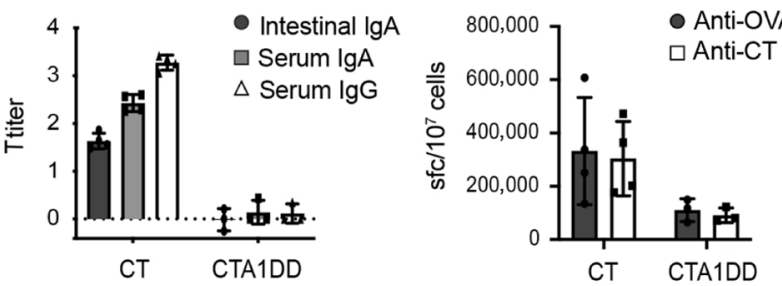

Fig. 5 Effects on TFH and GC B-cell responses in neonatal mice following oral priming immunization with CTA1-DD adjuvant. Neonatal mice were immunized on day 5 by feeding $5 \mu \mathrm{g}$ NP-OVA with or without $10 \mu \mathrm{g}$ CTA1-DD and boosted 30 days later with $5 \mu \mathrm{g}$ NP-OVA administered via oral gavage. The induction of GC B cells and TFH cells was assessed in the PPs at 10 days after priming (a, c) or in mesenteric lymph nodes (MLN), PPs, or the sub-mandibular lymph node $(\mathrm{smLN})$ at 5 days after an NP-OVA booster dose. $\mathbf{b}$, $\mathbf{d}-\mathbf{h}$ Flowcytometric analysis of the frequency and absolute number of GC B cells after priming (a) and after a booster immunization (b) and TFH cells after priming (c) and after a booster immunization (d). e Flowcytometric analysis of the percentage of GC B cells in the PP expressing Ephrin-B1 (EBI) or CD73 and $\mathbf{f}$ the frequency and number of plasma cells $\left(\mathrm{CD} 138^{+}\right)$in the PP induced by CTA1-DD adjuvant. $g$ Enhanced high-affinity NP-specific antibodies are found in serum from CTA1-DD adjuvanted immune responses. The mean ratio \pm SD is given for $\log _{10}$ titers of high-affinity (NP4) over lowaffinity (NP30) IgG antibodies. $\mathbf{h}$ Flowcytometric analysis of the percentage of lgA ${ }^{+}$GC B cells in the MLN and PP or CD138 ${ }^{+}$plasma cells in the PP following CTA1-DD adjuvant (black bar and +) or in unadjuvanted responses (gray bar and -). i Lack of adjuvant effect of CTA1-DD in adult mice following oral immunization. OVA-specific intestinal IgA, serum $\lg A$ or $\lg G$, and OVA- or CT-specific spot forming cells (SFC) in the lamina propria (LP) after 10 days following p.o. immunizations $(3 \times)$ of adult mice with $10 \mathrm{mg}$ OVA in the presence of cholera toxin (CT) or CTA1-DD adjuvant. All graphs are based on pooled data from 2-3 independent experiments with 2-5 mice in each group and results are given as means \pm SD. A statistical analysis was performed using two-way ANOVA followed by Dunnet correction for multiple comparison; ${ }^{*} p<0.05$, ${ }^{* *} p<0.01, * * * 0<0.001,{ }^{* * * *} p<0.0001$

lung $\mathrm{CD}^{+}{ }^{+} \mathrm{T}$ cells, attesting to the critical role of the CTA1 enzyme for the adjuvant effect (Fig. 6f).

\section{DISCUSSION}

The development of neonatal vaccination programs has been hampered partly by a lack of effective adjuvants that can mature and promote the innate immune system of neonates and young infants. ${ }^{10,12,13}$ Whereas in recent years some adjuvants have been described to promote neonatal GC formation, their mechanisms of action have been insufficiently investigated to allow for a rational design of neonatal vaccines or identify which route of administration that would improve their immunogenicity., ${ }^{4,57}$ Using the $C D 21^{\mathrm{mT} / \mathrm{mG}}$ mouse, we clearly identified that mucosal vaccination of neonatal mice was effective and that PPs, as opposed to peripheral lymph nodes and spleen, carried mature FDCs. Peyer's patches are the major sites for gut mucosal GC reactions and induction of secretory $\lg \mathrm{A}$ responses and can be targeted via oral immunization. ${ }^{58}$ Therefore, we attempted oral immunizations and found that this route allowed for effective priming of an anti-influenza immune response that resulted in reduced morbidity upon a virus challenge infection. ${ }^{59}$

The adjuvant, CTA1-DD, was found to strongly promote FDC expansion and maturation, but above all it enhanced 
a

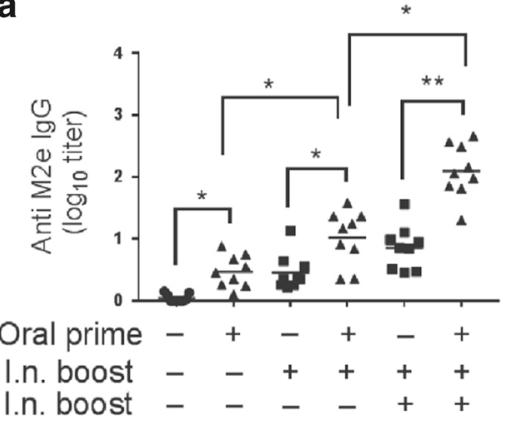

b
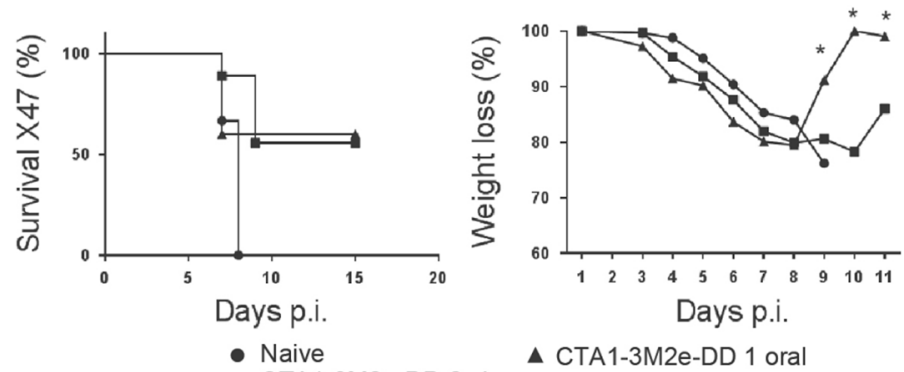

$\Delta$ CTA1-3M2e-DD 1 oral $+2 x$ i.n.

C
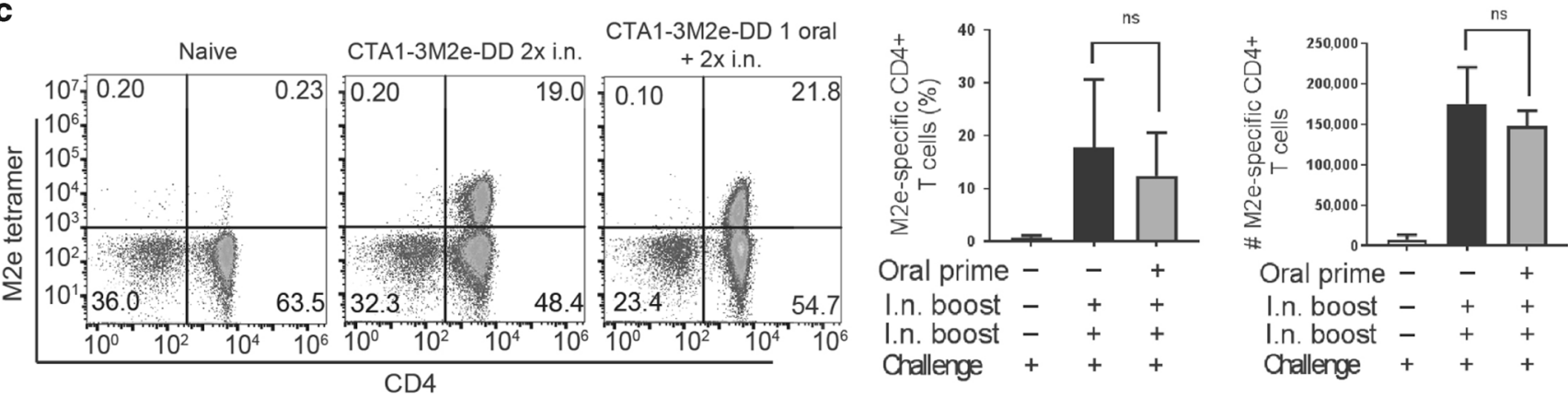

d

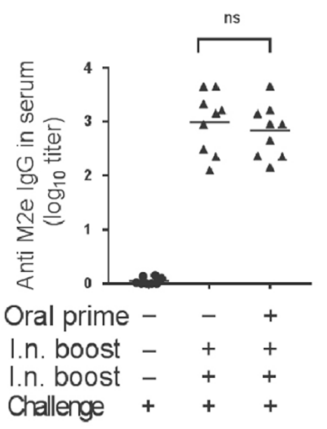

f

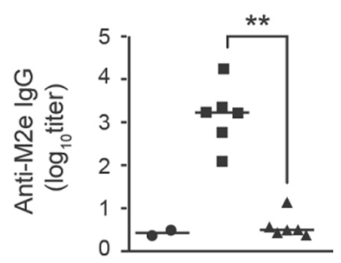

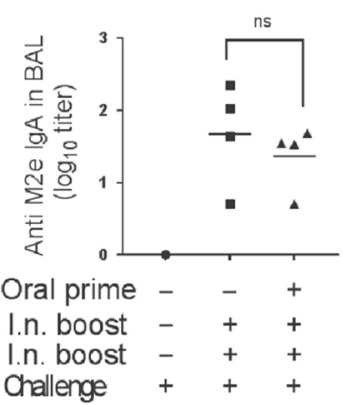

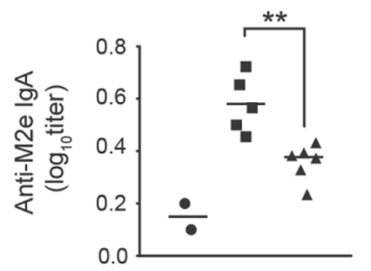

e
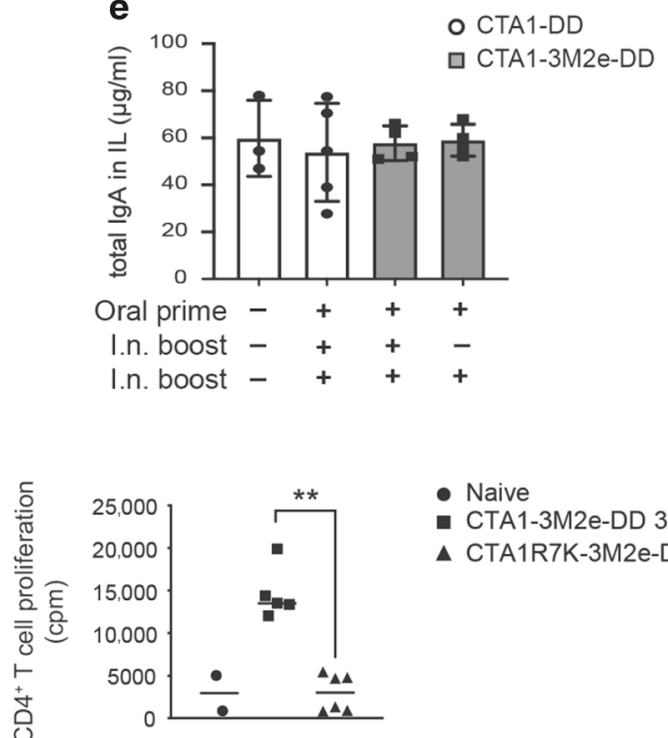

- Naive

- CTA1-3M2e-DD 3x i.n.

$\Delta$ CTA1R7K-3M2e-DD 3xi.n.

Fig. 6 Reduced morbidity to influenza infection in orally CTA1-3M2e-DD primed neonatal mice. Groups of ten mice were given PBS or primed p.o. with $10 \mu \mathrm{g}$ of CTA1-3M2e-DD followed by two intranasal immunizations with $10 \mu \mathrm{g}$ of CTA1-3M2e-DD and 15 days later the mice were challenged by $1 \times$ LD50 of a mouse adapted X47 influenza A virus strain. Neonatal mice were primed p.o. at post natal day 5 and received one or two i.n. booster doses with CTA1-3M2e-DD at 10-day intervals, as indicated. Control mice received only i.n immunizations with CTA1-3M2e-DD without the p.o. priming. a Anti-M2e IgG antibodies in serum were assessed after one, two, or three immunizations with CTA1-3M2e-DD without or with (d) a virus challenge infection. b Percentage of surviving animals and the monitored changes of body weight following an X47-challenge infection. c Representative FACS plots of M2e-tetramer-specific lung CD4 T cells isolated from mice immunized with CTA1-3M2e-DD, as indicated. Analysis of the frequency and absolute number M2e-specific CD4 T cells of all CD4 T cells in the lungs. d Anti-M2e IgG in serum and IgA in BAL after a challenge infection. e Total IgA in intestinal lavage after oral priming and i.n boosting with CTA1-3M2e-DD or CTA1-DD in neonatal mice, as indicated. $\mathbf{f}$ M2e-specific lgG and IgA titers in serum, and CD4 ${ }^{+}{ }^{-}$-cell proliferation to recall antigen in the mediastinal lymph node of adult mice following three i.n. doses of CTA1-3M2e-DD or the enzymatically inactive CTA1R7K-3M2e-DD. Statistical analysis was performed using two-way ANOVA followed by Dunnet correction for multiple comparison; ${ }^{*} p<0.05,{ }^{* *} p<0.01$.

transcription of GC-promoting genes, in particular, Cxcl13. Hence, a direct targeting of the vaccine-adjuvant formulation to FDCs improved the maturity and function, almost to adult levels, which resulted in significantly stronger Tfh and GC B-cell development.
This function appears to be different from that of other adjuvant formulations, of which most have been found to act through receptors on classical DCs. ${ }^{60}$ Moreover, the mechanism of action was dependent on the ADP-ribosylating ability of the CTA1- 
enzyme because the inactive mutant CTA1R7K-DD failed to enhance FDC functions. In a previous study we also demonstrated that complement activation by the DD-part enabled access to FDCs via their CR2s (CD21). ${ }^{22}$ By enhancing $\mathrm{Cr} 2$ gene expression in the FDC, CTA1-DD adjuvant could also influence CR2-mediated trapping of ICs to the FDC network, and, in this way increase antigen availability that could promote CSR and SHM in activated B cells. Thus, two different elements of the fusion protein, CTA1 and DD, contributed to its FDCimmunoenhancing functions.

The CD21-mT/mG mice were instrumental to the analysis of the FDC effects and by adding FDC-specific markers to our analysis, in particular FDC-M2, we were able to make clear distinctions between FDCs, non-FDC stromal cells and the GFP ${ }^{+} B$ cells. We found that CTA1-DD adjuvant bound to the FDC network in B-cell follicles of draining lymph nodes within hours of administration and subsequently within $24 \mathrm{~h}$ isolated FDCs exhibited enhanced gene expression of several GC-promoting genes, including the Cxcl13 gene, which encodes $\mathrm{Cxcl} 13$ that is the major lymphocyte chemokine attractant of activated Tfh and B cells to the GC. ${ }^{61}$ This effect also resulted in a marked increase in the size and frequency of GCs in the B-cell follicles. Noteworthy, because $\mathrm{Cxcl} 13$ has been reported to be a reliable biomarker of the $\mathrm{GC}$ reaction, ${ }^{36}$ correlating to enhanced antibody responses, we believe this effect of the adjuvant is central to its immunoenhancing functions. However, the effects on Icam-1 (26-fold), Cr2 (14-fold), II-6 (sixfold) and to a lesser extent $I-1 \beta, C d 40, L t \beta r$ and Baff gene expression were also enzyme-dependent and likely contributed to the effects on FDC maturation and the augmented GC-functions. ${ }^{22}$ These transcriptional changes were largely maintained over at least 4-6 days, which is the time when the GC reaction develops (reviewed in ref. ${ }^{62}$ ). The inactive mutant as well as the active CTA1-DD fusion protein also strongly enhanced IL-15 gene transcription, which could exert an anti-apoptotic effect on GC B cells in combination with CD40L-expressing Tfh cells. ${ }^{63}$ We convincingly demonstrated that CTA1-DD did not directly affect the activated $B$ cells using chimeric mice as we were able to exclude $B$ cells as targets of the CTA1-DD adjuvant action. CR2 expression in $\mathrm{B}$ cells had no influence on FDC gene expression, while CR-2 deficient FDC failed to show an upregulated $\mathrm{CxCl} 13$ gene expression.

The most unexpected finding of the present study was that CTA1-DD greatly impacted on immature FDCs in neonatal mice, resulting in their expansion and maturation and ability to strongly promote GC formations and isotype-switched antibody responses, almost to adult levels. More precisely, CTA1-DD adjuvant accelerated the kinetics of maturation of neonatal $\mathrm{CD} 21^{+} \mathrm{FDCs}$ and enhanced $\mathrm{CxCl} 13$ gene expression. While mature FDCs are not found in lymph nodes or spleen of neonatal mice the use of the $\mathrm{CD} 21^{\mathrm{mT} / \mathrm{mG}}$ mouse clearly identified and confirmed maturation of the FDC network following treatment with the CTA1-DD adjuvant. ${ }^{64}$ The effect was accompanied by increased frequencies of GC B cells and Tfh cells. Moreover, whereas the priming effect of CTA1-DD on GC development was prominent, a subsequent booster dose of antigen alone resulted in a dramatic augmentation of affinity matured antibody responses, suggesting that memory B cells, in particular, were favored by adjuvant treatment, which agrees well with previously published work in neonates. ${ }^{64}$ To this end, we observed that adjuvant enhanced Tfh cells in neonates expressed high levels of $\mathrm{Bcl}-6$, suggesting a strong influence on the GC reaction, which also resulted in long-lived plasma cell formation and an increased level of serum antibodies that was maintained for at least 5 weeks following a single priming immunization with CTA1-DD-adjuvant. Interestingly, following immunization with aluminum-adjuvanted vaccines earlier studies have suggested that the failure to develop TFH cells, in particular, is responsible for the impaired GC development in neonatal mice. ${ }^{65,66}$ It was suggested that both TFH-intrinsic and environmental factors fail in neonates requiring complementary stimuli to promote GC formations. ${ }^{40}$ Moreover, the related adjuvant, LTK63, has been reported to enhance GC reactions and specific antibody production in neonates through an effect on FDC maturation. ${ }^{37,39}$ By contrast, though, these effects appeared independent of gene transcriptional effects, but rather were dependent on marginal zone macrophages migrating into the GC and producing TNF. ${ }^{39}$ Indeed, LTK63 is largely lacking enzymatic activity and can thus be predicted not to influence gene transcription in FDCs. Other adjuvants have also been reported to affect the GC reaction. For example, MF59 improved GC formation in early life, but the effect depended on an enhanced TFH cell response rather than a direct effect on FDCs. ${ }^{15}$ As FDCs express TLRs, several other vaccine adjuvants may potentially activate these cells and the TLR4-stimuli LPS, for example, was found to increase CD16/32, ICAM- 1 and VCAM- 1 but this effect appeared only after 3 days, similar to the effects of Alum and MF59, and could be a consequence of tissue damage and inflammation, rather than an effect on FDCs. ${ }^{67,68}$ Thus, it appears that the CTA1-DD adjuvant is quite unique and effective at augmenting the $\mathrm{GC}$ reaction through a direct gene transcriptional effect on the FDC.

In mice, subsequent to establishing the microbiota there are considerable changes in the intestinal immune system in the first 14 weeks of life. ${ }^{69}$ Therefore, it is likely that oral immunizations are more effective early on than later in life when oral tolerance is the governing response pattern. ${ }^{51}$ Whereas, we could demonstrate in the present study as well in previously published work that CTA1DD is ineffective given orally to adult mice, it was apparent that its GC-promoting effects were excellent in 5-day-old mice. ${ }^{52}$ Indeed, we found that oral priming with CTA1-DD effectively targeted neonatal PPs, and significantly enhanced GC B-cell and TFH cell frequencies, leading to increased local $\lg \mathrm{A}^{+} \mathrm{B}$ cells in the PP and affinity matured serum IgG antibody responses. This fact, was further explored in the influenza vaccine model where we observed reduced morbidity and stronger serum IgG antibody responses in mice orally primed with CTA1-3M2e-DD compared to i.n. only immunization regimens. However, the oral CTA1-DD adjuvanted priming response did not stimulate a significant gut $\lg A$ response, arguing for multiple oral doses being required also in neonates for intestinal responses, which we have documented earlier for adult mice. ${ }^{59}$

We have recently shown that intranasal immunizations in adult mice using CTA1-3M2e-DD stimulated strong heterosubtypic protection against influenza infection, which relied heavily on M2e-specific resident memory CD4 T cells in the lung. ${ }^{70}$ Future studies are aimed to further investigate whether oral immunization using the CTA1-DD adjuvant is a way forward to allow for effective immunizations of neonates and young children. Earlier attempts at vaccinating against influenza in these age groups have been quite discouraging and associated with significant adverse reactions. ${ }^{71-74}$ However, these studies did not explore the potential of mucosal vaccination, but other vaccine studies have evaluated the use of oral priming in neonatal Macaque and Pig models against Simian immunodeficiency virus and rotavirus, albeit using live attenuated vaccines, with promising results. ${ }^{75,76}$ As oral priming is more effective for stimulating immune protection against enteric infections we also intend to test neonatal vaccination against rotavirus infections, using a CTA1VP6-DD fusion protein. ${ }^{76}$

\section{MATERIALS AND METHODS}

Mice and immunizations

C57BL/6 mice were obtained from Taconic (M\&B, Denmark) or Charles River (Kisslegg, Germany). CD21-Cre ${ }^{77}$ and Rosa ${ }^{\mathrm{mT} / \mathrm{mG}} 78$ mice (both obtained through $\mathrm{JAX}^{\circledR}$ Mice, USA) on a C57BL/6 background were crossed and the $\mathrm{F} 1$ generation $\left(\mathrm{CD} 21^{\mathrm{mT} / \mathrm{mG}}\right)$ was 
used for experiments. Mice were bred in ventilated cages at the Laboratory for Experimental Biomedicine, University of Gothenburg (Gothenburg, Sweden) and maintained under specific pathogen-free conditions. Age-matched mice were used throughout the study.

Germinal center formation and antibody responses were analyzed in mice immunized with $10 \mu \mathrm{g}$ CTA1-DD s.c. with $5 \mu \mathrm{g}$ NP-OVA, given in PBS with a total volume of $200 \mu \mathrm{l}$ (s.c.) or $20 \mu \mathrm{l}$ (i. n. and p.o.), respectively. Primary s.c. immunizations were followed by a s.c. booster dose of antigen only ( $5 \mu \mathrm{g}$ NP-OVA) on day 30. Neonatal immunizations were conducted at 5-7 days after birth with a total volume of $20 \mu$ s.c. or p.o. Serum samples for enzymelinked immune sorbent assay (ELISA) were taken on day 12 , day 28 , and day 35. Germinal center formations were analyzed 10 days (flow cytometry) or 12 days (IHC) after a primary immunization and 5 days (flow cytometry) after a booster immunization.

For tissue localization of labeled antigen/CTA1-DD, mice were given $20 \mu \mathrm{g}$ CTA1-DD i.v. or i.n. in $200 \mu \mathrm{l}$ or $20 \mu \mathrm{l} \mathrm{PBS,} \mathrm{respectively,}$ or $10 \mu \mathrm{g}$ CTA1-OVA-DD-AF488 in $200 \mu \mathrm{l} \mathrm{s.C.,} \mathrm{and} \mathrm{spleen,} \mathrm{mediast-}$ inal lymph nodes $(\mathrm{mLN})$ or inguinal lymph nodes (iLN) were removed at $6 \mathrm{~h}$ or $24 \mathrm{~h}$ after administration, as indicated.

For Influenza challenge studies, Balb/c mice were immunized orally 5 days after birth with $20 \mu \mathrm{l}$ PBS or PBS containing $5 \mu \mathrm{g}$ CTA1-3M2e-DD fusion protein and boosted two times intranasally (i.n.) at 4 and 6 weeks. All experiments were undertaken under the ethical permission 25/14 from the local ethical committee (Gothenburg, Sweden).

\section{Influenza virus challenge infection}

Two weeks after the last immunization, ten mice per group were challenged with live influenza A virus. A potentially lethal dose ( 2 or 1 LD50) of mouse adapted reassortant influenza A H3N2 virus strains X47 (A/Victoria/3/75 (H3N2) with A/Puerto Rico/8/34 (H1N1)) was diluted in PBS and administrated intranasally to mice lightly anesthetized by isofluoran solution. Mortality was monitored on a daily basis for 2 weeks after challenge. Morbidity was followed at 1-day intervals by monitoring body weight. According to the ethical permit mice were sacrificed when a loss of body weight exceeded $>30 \%$.

\section{Preparation of fusion proteins}

CTA1-DD, CTA1R7K-DD, CTA1-OVA-DD (carrying one copy of the immunodominant $\mathrm{OVA}_{323-339}$ peptide) or CTA1-3M2e-DD (carrying 3 copies of the influenza virus-specific M2e-peptide) were produced by Mivac Development AB (Gothenburg, Sweden) as described. ${ }^{79}$ Briefly, the fusion proteins were expressed in $E$. coli DH5 cells following transformation with the relevant expression vectors and produced as inclusion bodies. Fusion proteins were routinely tested for the presence of endotoxin, by end-point chromogenic limulus amebocyte lysate methods (LAL Endochrome $^{\mathrm{TM}}$, Charles River, Massachusetts, USA). Endotoxin-levels were consistently $<100$ endotoxin units/mg $(\mathrm{EU} / \mathrm{mg})$.

\section{Immunofluorescence}

Mediastinal lymph nodes ( $\mathrm{mLN})$, Peyer's patches (PP), or inguinal lymph nodes (iLN) were snap-frozen in OTC-embedding medium (Sakura Finetek, The Netherlands). Tissue sections $(7 \mu \mathrm{m})$ were prepared on micro-slides using a Zeiss cryostat (Carl Zeiss AG. Germany). Sections were air dried, fixed in acetone and blocked using $5 \%$ horse serum and then stained by immunofluorescence against the antigen of interest at $4{ }^{\circ} \mathrm{C}$ (Supplementary Table 1). CTA1-DD was identified by a chicken anti-CTA1-DD antibody (Agrisera, Sweden) in combination with goat anti-chickenDyLight488 or as directly AF488-labeled CTA1-OVA-DD fusion protein (Antibody labeling kit, Thermo Fisher Scientific, USA).

Confocal microscopy was performed at the Center for cellular Imaging (CCl, University of Gothenburg, Sweden) using the Zeiss LSM 510 META system and LSM software (Carl Zeiss, AG,
Germany). All images within an experiment were equivalently manipulated using Zen software (Carl Zeiss AG, Germany.) to adjust brightness and contrast. For quantification of color intensity, images were imported into Fiji, ${ }^{80}$ transformed into gray-scale, B-cell follicles were defined as region of interest and the mean color intensity within the region was measured.

\section{Flow cytometry detection of GC B cells and TFH}

Lymph nodes were forced through a nylon mesh using a syringe plunger. Peyer's patches were washed with $2 \mathrm{mM}$ EDTA for $30 \mathrm{~min}$ at $37^{\circ} \mathrm{C}$ and then digested with Collagenase $\mathrm{D}(0.5 \mathrm{U} / \mathrm{ml}$ final) for $1 \mathrm{~h}$ at $37^{\circ} \mathrm{C}$. Supernatant and remaining tissue pieces were forced through a nylon mesh using a syringe plunger. Cells were stained with fixable viability die Aqua for $30 \mathrm{~min}$ at $4{ }^{\circ} \mathrm{C}$, washed and then incubated with fluorescently labeled antibodies for surface antigens for $30 \mathrm{~min}$ at $4{ }^{\circ} \mathrm{C}$ (see Supplementary Table 1). Two separate panels were used for detection of GC B cells and TFH cells, respectively. Intracellular labeling for FoxP3 and $\mathrm{Bcl}-6$ transcription factors was performed after fixation of cells for 30 min at $4{ }^{\circ} \mathrm{C}$ using the FoxP3 Transcription Factor Fixation/ Permeabilization kit (eBioscience, USA). After labeling, cells were washed twice and analyzed using a BD-FACS LSR II instrument (BD Bioscience, USA) or a CytoFLEX flow cytometer (Beckman Coulter, USA) and data analyzed with FlowJo (TreeStar, USA) software.

\section{M2e-tetramer staining}

Two weeks after challenge, lung cells were dissociated using Lung Dissociation Kit (Miltenyi Biotec Norden AB, Sweden), according to the manufacturer's instructions. To assess the presence of $M 2 \mathrm{e}-$ specific $\mathrm{CD}^{+}{ }^{+} \mathrm{T}$ cells, lung cells were stained with $\mathrm{M} 2 \mathrm{e}$-tetramer-PE (NIH Tetramer core facility, USA) at $37^{\circ} \mathrm{C}$ for $30 \mathrm{~min}$ and then incubated with fluorescently labeled antibodies for surface antigens at $4{ }^{\circ} \mathrm{C}$ for $30 \mathrm{~min}$ (all from eBiosciences, USA). In all, $1,000,000$ events were acquired on a BD-FACS LSR II instrument (BD Bioscience, USA) and data analyzed with FlowJo (TreeStar, USA) software. Thresholds of positivity were identified using fluorescence minus one control for each color on cells from each sample group. For proliferation analysis, single cell suspensions $(2 \times 106 \mathrm{cells} / \mathrm{ml})$ from lung from immunized and control mice were cultured for in vitro M2e-peptide recall responses in triplicates in 96-well microtiter plates (Nunc, Denmark) for $72 \mathrm{~h}$ at $37^{\circ} \mathrm{C}$ in $5 \% \mathrm{CO} 2$. After $72 \mathrm{~h}$, we added [3 H]-thymidine (PerkinElmer, USA) to the cultures for the last $6 \mathrm{~h}$ and $[3 \mathrm{H}]-$ thymidine uptake was determined using a scintillation counter (Beckman, Sweden).

Anti-NP and M2e antibodies in serum, broncheoalveolar lavage (BAL), and intestinal lavage (IL)

Antibody titers were measured using ELISA (Enzyme-Linked ImmunoSorbent Assay). Briefly, 96-well microtiter plates (Maxisorp, Nunc, Denmark) were coated with $10 \mu \mathrm{g} / \mathrm{ml}$ of NP4-BSA (Biosearch technologies, California, USA) or $2 \mu \mathrm{g} / \mathrm{ml}$ of $\mathrm{M} 2 \mathrm{e}$ pepide in PBS at $4{ }^{\circ} \mathrm{C}$ overnight. After washing with PBS and blocking with $0.1 \%$ $\mathrm{BSA} / \mathrm{PBS}$ for $30 \mathrm{~min}$ at $37^{\circ} \mathrm{C}$, serum samples were diluted $1 / 50$ or $1 / 25$ in $0.1 \%$ BSA/PBS and loaded in the wells. BAL and IL samples were diluted to $1 / 2$. A series of $1 / 3$ dilutions of the different serum samples in corresponding subwells were performed. Plates were then incubated for $2 \mathrm{~h}$ at r.t. After washing with PBS/Tween, alkaline phosphatase-conjugated goat anti-mouse $\lg G$ tot, $\lg G 1$, IgG2a, or IgG2c Abs (Southern Biotechnology Associates, Inc., USA) were added to the wells, diluted $1 / 500$ in $0.1 \%$ BSA/PBS. Plates were incubated for $2 \mathrm{~h}$ at r.t. Ag-specific serum reactivity was visualized using phosphatase substrate tablets, nitrophenyl disodium salt hexahydrate (Sigma-Aldrich, Germany) dissolved at $1 \mathrm{mg} / \mathrm{ml}$ in ethanolamine buffer $(\mathrm{pH} 9.8)$ were added to the wells. Absorbance was measured at $405 \mathrm{~nm}$ using a Multiscan MS spectrophotometer (USA). The linear part of the curve was used for calculating $\log _{10}$-titers at a cutoff value of 0.4 . 
Cell sorting

For analysis of FDC and B-cell gene expression, CD21-Cre GFP ${ }^{+}$FDCs or B cells were isolated as described before. ${ }^{32,44}$ Lymph nodes were digested for $30 \mathrm{~min}$ with a mixture of Liberase TL $(1.3 \mathrm{U} / \mathrm{ml}$, Roche, Switzerland) and DNase I ( $50 \mathrm{U} / \mathrm{ml}$, Roche, Switzerland), followed by two washing steps with ice-cold PBS with $0.5 \%$ BSA and 2 mM EDTA on ice. Before antibody labeling, cells were treated with FcR-blocking antibody (2.4G2) for $15 \mathrm{~min}$. Lymphocytes were labeled with fluorochrome conjugated antibodies specific for surface antigens (CD45-APCCy7, CD31-BV605, FDC-M2 biotin + StAv-BV421, gp38 PECy7, dump: tdTomato, Ter119-PE, GR1-PE, CD11C-PE, F4/80-PE, CD138-PE) and incubated with live/dead stain 7-AAD for $10 \mathrm{~min}$ on ice. Single cells, negative for 7-AAD, dump, CD31 and CD45, with positive staining for MGFP and FDC-M2 were sorted using a BD Aria III flow cytometer (BD, Germany) to $>85 \%$ purity.

RNA extraction and quantitative real-time PCR

FDC sorted from whole LNs were collected and resuspended in RLT buffer (Qiagen, Germany). Total RNA was extracted using RNeasy Mini kit (Qiagen, Germany), genomic DNA was eliminated using a DNase treatment step according to the manufacturer's instructions. cDNA was made using the QuantiTect reverse transcription kit (Qiagen, Germany) from $100 \mathrm{ng}$ total RNA (2-3 samples were pooled if RNA yield/mouse was too low). Samples were loaded onto a custom RT2 Profiler PCR 96-well array plate (SABiosciences, Germany), and RT-qPCR was performed on a light cycler 480 system (Roche) (Supplementary Table 2). Gene expression fold-change was calculated as $2^{\text {ddct }}$ relative to Hprt and Gapdh as reference gene and mRNA from unimmunized cells.

Statistical analysis

The analyses were performed using the Prism software (GraphPad). All reported $p$-values are two-sided and values of $<0.05$ were considered to indicate statistical significance. $p<0.05(*), p<0.01$ $\left.{ }^{(* *)}, p<0.001{ }^{(* *}\right)$.

\section{DATA AVAILABILITY}

The authors declare that the data supporting the findings of this study are available within the article and its Supplementary Information files, or are available from the authors upon request.

\section{ACKNOWLEDGEMENTS}

We thank professor Claire-Ann Siegrist (WHO collaborating Center of Vaccinology \& Neonatal Immunology, Geneva) for introducing us to neonatal vaccination and how to work with the neonatal mouse model. This study was supported in parts by research funds from the Knut and Alice Wallenberg Foundation KAW 2013.0030, Swedish Foundation for Strategic Research SB12-0088, The Swedish Cancer Foundation, The Swedish Research Council, EU projects in FP7 UniVacFlu and UNISEC, LUA/ALF ALFGBG-531021 and the Lundberg foundation. We also thank the Center for Cellular Imaging and the Laboratory for Experimental Biomedicine Animal Facility at the University of Gothenburg.

\section{AUTHOR CONTRIBUTIONS}

S.S., J.M., V.B., and N.L. designed the study, analyzed data, and wrote the manuscript. S.S., V.B., U.W., A.S., and K.S., did the experiments and analysis of the data, performed flow cytometry, molecular biology analysis, and confocal microscopy. S.S. designed the qPCR experimental set-up and analysis. V.B. and K.S. conducted the Influenza challenge studies.

\section{ADDITIONAL INFORMATION}

The online version of this article (https://doi.org/10.1038/s41385-020-0253-2) contains supplementary material, which is available to authorized users.

Competing interests: The authors declare no competing interests.
Publisher's note Springer Nature remains neutral with regard to jurisdictional claims in published maps and institutional affiliations.

\section{REFERENCES}

1. Kollmann, T. R., Kampmann, B., Mazmanian, S. K., Marchant, A. \& Levy, O. Protecting the newborn and young infant from infectious diseases: lessons from immune ontogeny. Immunity 46, 350-363 (2017).

2. Zhang, X., Zhivaki, D. \& Lo-Man, R. Unique aspects of the perinatal immune system. Nat. Rev. Immunol. 17, 495-507 (2017).

3. Saso, A. \& Kampmann, B. Vaccine responses in newborns. Semin. Immunopathol. 39, 627-642 (2017).

4. Morris, M. C. \& Surendran, N. Neonatal vaccination: challenges and intervention strategies. Neonatology 109, 161-169 (2016).

5. Kilich, E. \& Anthony, M. Rotavirus vaccination in preterm infants: a neonatal guidance chart to aid timely immunisation. Arch. Dis. Child. Fetal Neonatal Ed. 100, F465 (2015).

6. Halsey, N. \& Galazka, A. The efficacy of DPT and oral poliomyelitis immunization schedules initiated from birth to 12 weeks of age. Bull. World Health Organ. 63, 1151-1169 (1985).

7. Saleh, E. et al. Open-Label pilot study to compare the safety and immunogenicity of pentavalent rotavirus vaccine (RV5) administered on an early alternative dosing schedule with those of RV5 administered on the recommended standard schedule. J. Pediatr. Infect. Dis. Soc. 7, 82-85 (2018).

8. Mohr, E. \& Siegrist, C. A. Vaccination in early life: Standing up to the challenges. Curr. Opin. Immunol. 41, 1-8 (2016).

9. Basha, S., Surendran, N. \& Pichichero, M. Immune responses in neonates. Expert Rev. Clin. Immunol. 10, 1171-1184 (2014).

10. Kampmann, B. \& Jones, C. E. Factors influencing innate immunity and vaccine responses in infancy. Philos. Trans. R. Soc. B. Biol. Sci. 370, 1-5 (2015).

11. Mastelic, B. et al. Environmental and T cell-intrinsic factors limit the expansion of neonatal follicular T helper cells but may be circumvented by specific adjuvants. J. Immunol. 189, 5764-5772 (2012).

12. Levy, O. Innate immunity of the newborn: basic mechanisms and clinical correlates. Nat. Rev. Immunol. 7, 379-390 (2007).

13. Zaghouani, H., Hoeman, C. M. \& Adkins, B. Neonatal immunity: faulty T-helpers and the shortcomings of dendritic cells. Trends Immunol. 30, 585-591 (2009).

14. Marrack, P., McKee, A. S. \& Munks, M. W. Towards an understanding of the adjuvant action of aluminium. Nat. Rev. Immunol. 9, 287-293 (2009).

15. Mastelic Gavillet, B. et al. MF59 mediates Its B cell adjuvanticity by promoting T follicular helper cells and thus germinal center responses in adult and early life. $J$. Immunol. 194, 4836-4845 (2015).

16. He, P., Zou, Y. \& Hu, Z. Advances in aluminum hydroxide-based adjuvant research and its mechanism. Hum. Vaccines Immunother. 11, 477-488 (2015).

17. Agren, L. C., Ekman, L., Lowenadler, B. \& Lycke, N. Y. Genetically engineered nontoxic vaccine adjuvant that combines $B$ cell targeting with immunomodulation by cholera toxin A1 subunit. J. Immunol. 158, 3936-3946 (1997).

18. Eriksson, A. M., Schon, K. M. \& Lycke, N. Y. The cholera toxin-derived CTA1-DD vaccine adjuvant administered intranasally does not cause inflammation or accumulate in the nervous tissues. J. Immunol. 173, 3310-3319 (2004).

19. Helgeby, A. et al. The combined CTA1-DD/ISCOM adjuvant vector promotes priming of mucosal and systemic immunity to incorporated antigens by specific targeting of B cells. J. Immunol. 176, 3697-3706 (2006).

20. Agren, L. et al. The ADP-ribosylating CTA1-DD adjuvant enhances $T$ celldependent and independent responses by direct action on $B$ cells involving antiapoptotic Bcl-2- and germinal center-promoting effects. J. Immunol. 164, 6276-6286 (2000)

21. Bemark, $M$. et al. A unique role of the cholera toxin A1-DD adjuvant for long-term plasma and memory B cell development. J. Immunol. 186, 1399-1410 (2011).

22. Mattsson, J. et al. Complement activation and complement receptors on follicular dendritic cells are critical for the function of a targeted adjuvant. J. Immunol. 187, 3641-3652 (2011)

23. Park, C. S. \& Choi, Y. S. How do follicular dendritic cells interact intimately with B cells in the germinal centre? Immunology 114, 2-10 (2005).

24. Bajenoff, M. \& Germain, R. N. B-cell follicle development remodels the conduit system and allows soluble antigen delivery to follicular dendritic cells. Blood 114, 4989-4997 (2009).

25. Phan, T. G., Green, J. A., Gray, E. E., Xu, Y. \& Cyster, J. G. Immune complex relay by subcapsular sinus macrophages and noncognate $B$ cells drives antibody affinity maturation. Nat. Immunol. 10, 786-793 (2009).

26. Vora, K. A., Ravetch, J. V. \& Manser, T. Amplified follicular immune complex deposition in mice lacking the Fc receptor gamma-chain does not alter maturation of the B cell response. J. Immunol. 159, 2116-2124 (1997). 
27. Hannum, L. G., Haberman, A. M., Anderson, S. M. \& Shlomchik, M. J. Germinal center initiation, variable gene region hypermutation, and mutant $B$ cell selection without detectable immune complexes on follicular dendritic cells. J. Exp. Med 192, 931-942 (2000).

28. Allen, C. D. \& Cyster, J. G. Follicular dendritic cell networks of primary follicles and germinal centers: phenotype and function. Semin Immunol. 20, 14-25 (2008).

29. Suzuki, K., Grigorova, I., Phan, T. G., Kelly, L. M. \& Cyster, J. G. Visualizing B cell capture of cognate antigen from follicular dendritic cells. J. Exp. Med 206, 1485-1493 (2009).

30. Wang, X. et al. Follicular dendritic cells help establish follicle identity and promote B cell retention in germinal centers. J. Exp. Med 208, 2497-2510 (2011).

31. Roozendaal, R. et al. Conduits mediate transport of low-molecular-weight antigen to lymph node follicles. Immunity 30, 264-276 (2009).

32. Suzuki, K. et al. The sensing of environmental stimuli by follicular dendritic cells promotes immunoglobulin A generation in the gut. Immunity 33, 71-83 (2010).

33. Ansel, K. M. et al. A chemokine-driven positive feedback loop organizes lymphoid follicles. Nature 406, 309-314 (2000).

34. Allen, C. D., Okada, T. \& Cyster, J. G. Germinal-center organization and cellular dynamics. Immunity 27, 190-202 (2007).

35. Finch, D. K., Ettinger, R., Karnell, J. L., Herbst, R. \& Sleeman, M. A. Effects of CXCL13 inhibition on lymphoid follicles in models of autoimmune disease. Eur. J. Clin. Invest 43, 501-509 (2013).

36. Havenar-Daughton, C. et al. CXCL13 is a plasma biomarker of germinal center activity. Proc. Natl Acad. Sci. USA 113, 2702-2707 (2016).

37. Aradottir Pind, A. A. et al. Adjuvants enhance the induction of germinal center and antibody secreting cells in spleen and their persistence in bone marrow of neonatal mice. Front. Immunol. 10, 1-13 (2019).

38. Cantisani, R. et al. Vaccine adjuvant MF59 promotes retention of unprocessed antigen in lymph node macrophage compartments and follicular dendritic cells. J. Immunol. 194, 1717-1725 (2015).

39. Bjarnarson, S. P., Adarna, B. C., Benonisson, H., Giudice, G. D. \& Jonsdottir, I. The adjuvant LT-K63 can restore delayed maturation of follicular dendritic cells and poor persistence of both protein- and polysaccharide-specific antibody-secreting cells in neonatal mice. J. Immunol. 189, 1265-1273 (2012).

40. Vono, M. et al. Overcoming the neonatal limitations of inducing germinal centers through liposome-based adjuvants including C-type lectin agonists trehalose dibehenate or Curdlan. Front. Immunol. 9, 1-12 (2018).

41. Mionnet, $C$. et al. Identification of a new stromal cell type involved in the regulation of inflamed B cell follicles. PLoS Biol. 11, e1001672 (2013).

42. Rodda, L. B., Bannard, O., Ludewig, B., Nagasawa, T. \& Cyster, J. G. Phenotypic and morphological properties of germinal center dark zone Cxcl12-expressing reticular cells. J. Immunol. 195, 4781-4791 (2015).

43. Sitnik, K. M. et al. Context-dependent development of lymphoid stroma from adult CD34+adventitial progenitors. Cell Rep. 14, 2375-2388 (2016).

44. Sukumar, S., Szakal, A. K. \& Tew, J. G. Isolation of functionally active murine follicular dendritic cells. J. Immunol. Methods 313, 81-95 (2006).

45. Pihlgren, $M$. et al. Unresponsiveness to lymphoid-mediated signals at the neonatal follicular dendritic cell precursor level contributes to delayed germinal center induction and limitations of neonatal antibody responses to T-dependent antigens. J. Immunol. 170, 2824-2832 (2003).

46. Munguia-Fuentes, R. et al. Immunization of newborn mice accelerates the architectural maturation of lymph nodes, but AID-dependent IgG responses are still delayed compared to the adult. Front Immunol. 8, 13 (2017).

47. Cupedo, T. et al. Initiation of cellular organization in lymph nodes is regulated by non-B cell-derived signals and is not dependent on CXC chemokine ligand 13. J. Immunol. 173, 4889-4896 (2004).

48. Chen, D., Hoshi, H., Tanaka, K. \& Murakami, G. Postnatal development of lymphoid follicles in rat Peyer's patches, with special reference to increased follicle number. Arch. Histol. Cytol. 58, 335-343 (1995).

49. Hoshi, H. et al. Patterns of age-dependent changes in the numbers of lymph follicles and germinal centres in somatic and mesenteric lymph nodes in growing C57B1/6 mice. J. Anat. 198, 189-205 (2001).

50. Reboldi, A., Cyster, J. G., Francisco, C. S. \& Francisco, S. Peyer's patches: organizing B-cell responses at the intestinal frontier. Immunol Rev. 271, 230-245 (2017).

51. Strobel, S. Neonatal oral tolerance. Ann. N. Y. Acad. Sci. 778, 88-102 (1996).

52. Mowat, A. M. I. et al. CTA1-DD-immune stimulating complexes: a novel, rationally designed combined mucosal vaccine adjuvant effective with nanogram doses of antigen. J. Immunol. 167, 3398-3405 (2001).

53. Laidlaw, B. J. et al. The Eph-related tyrosine kinase ligand Ephrin-B1 marks germinal center and memory precursor B cells. J. Exp. Med. https://doi.org/ 10.1084/jem.20161461 (2017). jem.20161461.
54. Conter, L. J., Song, E., Shlomchik, M. J. \& Tomayko, M. M. CD73 expression is dynamically regulated in the germinal center and bone marrow plasma cells are diminished in its absence. PLOS ONE 9, e92009 (2014).

55. Weisel, F. J., Zuccarino-Catania, G. V., Chikina, M. \& Shlomchik, M. J. A temporal switch in the germinal center determines differential output of memory $B$ and plasma cells. Immunity 44, 116-130 (2016).

56. Taylor, J. J., Pape, K. A. \& Jenkins, M. K. A germinal center-independent pathway generates unswitched memory $\mathrm{B}$ cells early in the primary response. J. Exp. Med. 209, 597-606 (2012).

57. Savelkoul, H. F. J., Ferro, V. A., Strioga, M. M. \& Schijns, V. E. J. C. Choice and design of adjuvants for parenteral and mucosal vaccines. Vaccines 3, 148-171 (2015).

58. Mestecky, J. \& Elson, C. O. Peyer's Patches as the Inductive Site for IgA Responses. J. Immunol. 180, 1293-1294 (2008).

59. Lycke, N. Recent progress in mucosal vaccine development: potential and limitations. Nat. Rev. Immunol. 12, 592-605 (2012).

60. Chappell, C. P., Giltiay, N. V., Dresch, C. \& Clark, E. A. Controlling immune responses by targeting antigens to dendritic cell subsets and B cells. Int Immunol. 26, 3-11 (2014).

61. Okada, T. et al. Chemokine requirements for B cell entry to lymph nodes and Peyer's patches. J. Exp. Med 196, 65-75 (2002).

62. Silva, N. S. D. \& Klein, U. Dynamics of B cells in germinal centres. Nat. Rev. Immunol. 15, 137-148 (2015).

63. Epron, G. et al. Monocytes and T cells cooperate to favor normal and follicular lymphoma B-cell growth: role of IL-15 and CD40L signaling. Leukemia 26, 139-148 (2012).

64. Siegrist, C. A. \& Aspinall, R. B-cell responses to vaccination at the extremes of age. Nat. Rev. Immunol. 9, 185-194 (2009).

65. Mastelic, B. et al. Environmental and T cell-intrinsic factors limit the expansion of neonatal follicular T helper cells but may be circumvented by specific adjuvants. J. Immunol. 189, 5764-5772 (2012).

66. Debock, l. et al. Neonatal follicular Th cell responses are impaired and modulated by IL-4. J. Immunol. 191, 1231-1239 (2013).

67. Aydar, Y., Balogh, P., Tew, J. G. \& Szakal, A. K. Altered regulation of Fc gamma RII on aged follicular dendritic cells correlates with immunoreceptor tyrosine-based inhibition motif signaling in B cells and reduced germinal center formation. $J$. Immunol. 171, 5975-5987 (2003).

68. Cantisani, R. \& Piccioli, D. Vaccine adjuvants confer an advantage to the kinetics of activation of follicular dendritic cells that are sensitive to peripheral tissue's injury. Scand. J. Immunol. 82, 144-146 (2015).

69. Gensollen, T., lyer, S. S., Kasper, D. L., Blumberg, R. S. \& Medical, H. How colonization by microbiota in early life shapes the immune system. Science $\mathbf{3 5 2}$ 539-544 (2016).

70. Eliasson, D. G. et al. CTA1-M2e-DD: a novel mucosal adjuvant targeted influenza vaccine. Vaccine 26, 1243-1252 (2008).

71. Kotloff, K. L. et al. Clinical and immune responses to inactivated influenza A (H1N1)pdm09 vaccine in children. Pediatr. Infect. Dis. J. 33, 865-871 (2014).

72. Shinjoh, M. et al. Effectiveness of trivalent inactivated influenza vaccine in children estimated by a test-negative case-control design study based on influenza rapid diagnostic test results. PLOS ONE 10, 1-15 (2015).

73. Nakaya, H. I. et al. Systems biology of immunity to MF59-adjuvanted versus nonadjuvanted trivalent seasonal influenza vaccines in early childhood. Proc. Natl Acad. Sci. USA 113, 1853-1858 (2016)

74. Belshe, R. B. et al. Live attenuated versus inactivated influenza vaccine in infants and young children. N. Engl. J. Med. 685-696 (2007). https://doi.org/10.1056/ NEJMoa065368.

75. Jensen, K. NIH Public Access. 53-63 (2013). https://doi.org/10.1016/j.trivac.2013. 09.005 .

76. Azevedo, M. S. P. et al. An oral versus intranasal prime/boost regimen using attenuated human rotavirus or VP2 and VP6 virus-like particles with immunostimulating complexes influences protection and antibody-secreting cell responses to rotavirus in a neonatal gnotobiotic pig mode. Clin. Vaccin. Immunol. 17, 420-428 (2010).

77. Kraus, M., Alimzhanov, M. B. \& Rajewsky, N. Survival of resting mature B lymphocytes depends on BCR signaling via the Igalpha/beta heterodimer. Cell 117, 787-800 (2004).

78. Muzumdar, M. D., Tasic, B., Miyamichi, K., Li, L. \& Luo, L. A global doubleflourescent Cre reporter mouse. Genesis 224, 219-224 (2006).

79. Agren, L. C., Ekman, L., Lowenadler, B., Nedrud, J. G. \& Lycke, N. Y. Adjuvanticity of the cholera toxin A1-based gene fusion protein, CTA1-DD, is critically dependent on the ADP-ribosyltransferase and lg-binding activity. J. Immunol. 162, 2432-2440 (1999).

80. Schindelin, J. et al. Fiji: an open-source platform for biological-image analysis. Nat. Methods 9, 676 (2012). 